\title{
Separating valid odd-cycle and odd-set inequalities for the multiple depot vehicle scheduling problem
}

\author{
Mounira Groiez • Guy Desaulniers • \\ Ahmed Hadjar • Odile Marcotte
}

Received: 14 December 2012 / Accepted: 22 June 2013 / Published online: 24 July 2013

(C) Springer-Verlag Berlin Heidelberg and EURO - The Association of European Operational Research Societies 2013

\begin{abstract}
We consider the multicommodity network flow formulation of the multiple depot vehicle scheduling problem (MDVSP) and investigate several strategies within a branch-and-cut framework for solving the MDVSP. In particular, we compare subalgorithms for separating the cutting planes introduced by Hadjar et al. (Oper Res 54:130-149, 2006). We also evaluate the effectiveness of a new criterion for fixing some variables in the multicommodity flow formulation. Finally, we present computational experiments to illustrate the strengths and weaknesses of the strategies.
\end{abstract}

Keywords Transportation scheduling $\cdot$ Multicommodity flow $\cdot$ Branch-and-cut algorithm

Mathematics Subject Classification (2000) 90C27 · 90C57 · $90 \mathrm{~B} 06$

\footnotetext{
M. Groiez

Département de mathématiques et génie industriel, École Polytechnique de Montréal, C.P. 6079, succ. Centre-ville, Montreal H3C 3A7, Canada

G. Desaulniers

Département de mathématiques et génie industriel, École Polytechnique de Montréal, and GERAD, C.P. 6079, succ. Centre-ville, Montreal H3C 3A7, Canada
}

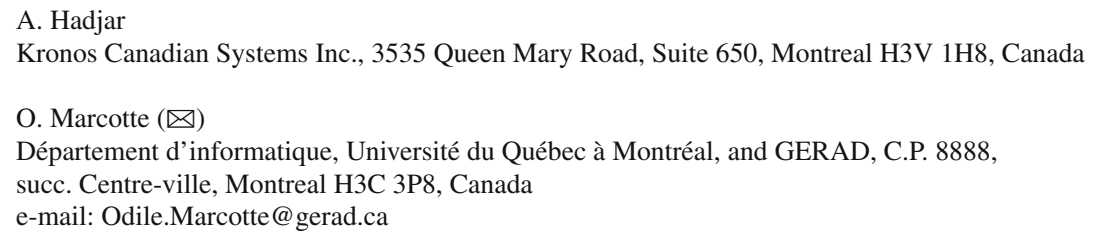




\section{Introduction}

In the vast area of time-constrained routing and scheduling, one of the most studied problems is the multiple depot vehicle scheduling problem (MDVSP), in which one looks for a minimum-cost assignment of trips to vehicles satisfying some precedence constraints. The MDVSP was first introduced in Bodin et al. (1978) and proved to be NP-complete by Bertossi et al. (1987). It can be formulated in several ways as an integer programming problem and solved by exact methods including branch-andbound, column generation, Lagrangian relaxation, and cutting planes. For instance, Bianco et al. (1994) have proposed a set partitioning approach to the MDVSP. Fischetti et al. (2001) used the single-commodity flow model to formulate the MDVSP as an integer program; the latter has a large number of constraints and they also introduced families of valid inequalities for their formulation.

Löbel (1998) proposed an algorithm based on Lagrangian relaxation for solving the MDVSP. Ribeiro and Soumis (1994) introduced column generation for solving the MDVSP and their article was followed by several articles using this paradigm (see in particular Hadjar et al. 2006; Oukil et al. 2007). Gintner et al. (2005) have proposed a two-phase method producing close to optimal solutions for instances consisting of thousands of trips. Kliewer et al. (2006) were able to solve very large real-world instances by using time-space networks instead of the usual connection-based networks. Finally, some authors (Laurent and Hao 2009; Pepin et al. 2009) have studied heuristics for the MDVSP. Since there is a vast literature on the MDVSP, we refer the reader to the surveys by Desrosiers et al. (1995) and Desaulniers and Hickman (2007).

Hadjar et al. (2006) have studied the multicommodity flow model for the MDVSP and derived a large class of valid inequalities based on odd sets (henceforth called odd-set inequalities). For solving the linear relaxation of the MDVSP, they proposed an algorithm combining column generation and variable fixing. This algorithm is embedded into a branch-and-cut algorithm based on the explicit enumeration of some cycles giving rise to odd-set inequalities. Their results demonstrate that the cutting planes based on odd-set inequalities can reduce the solution time of MDVSP instances by as much as $50 \%$. The present article explores issues that were not addressed in Hadjar et al. (2006).

- What is the performance of the branch-and-cut algorithm described in Hadjar et al. (2006) if one does not use column generation but solves the linear relaxation of the multicommodity flow model instead?

- Is it worthwhile to use the bidirectional method for fixing variables presented in Irnich et al. (2010)?

- Can one generate cutting planes by a method that avoids enumeration of cycles?

In "Background" we define the MDVSP precisely, present the multicommodity flow model, and review the odd-set inequalities. In "Finding violated valid inequalities through a search of the conflict graph" we describe an algorithm for finding some violated valid inequalities by searching the conflict graph of the graph underlying an MDVSP instance. In "Finding violated valid inequalities through an auxiliary integer program" we present an integer programming approach to finding a broader class of violated valid inequalities than in "Finding violated valid inequalities through a 
search of the conflict graph". In "Variable fixing and strategies" we show how to fix variables by adapting a procedure described in Irnich et al. (2010) and propose various strategies for our branch-and-cut algorithm. Finally "Computational results" contains our experimental results and "Conclusion" our conclusion.

\section{Background}

In an instance of the MDVSP, one is given $n$ trips or tasks denoted by $T_{1}, T_{2}, \ldots, T_{n}$ and $|K|$ depots denoted by $D_{1}, D_{2}, \ldots, D_{|K|}$, where $K$ is an index set. The $n$ trips must be assigned to identical vehicles housed in the depots. The availability at depot $D_{k}$ is denoted by $v_{k}$; thus the number of vehicles leaving $D_{k}$ is at most $v_{k}$ (for every $k$ ). A route (or duty) is a sequence of trips that are assigned to a specific vehicle starting from depot $D_{k}$ (for some $k$ ) and returning to the same depot at the end of the sequence. In this sequence the trip $T_{i}$ is the predecessor of the trip $T_{j}$ if a vehicle can carry out $T_{j}$ immediately after $T_{i}$. To make this definition more precise and pave the way for the integer programming formulation, we introduce the directed multigraph $G=(V, A)$, defined as follows (see Hadjar et al. 2006). The vertex set of $G$ is the union of the sets $\left\{T_{1}, T_{2}, \ldots, T_{n}\right\}$ and $\left\{D_{1}, D_{2}, \ldots, D_{|K|}\right\}$. There are two kinds of $\operatorname{arcs}$ in $G$ : first the $\operatorname{arcs}\left(T_{i}, T_{j}, k\right)$, where $T_{j}$ can be carried out immediately after $T_{i}$ and $k$ is any index in $\{1,2, \ldots,|K|\}$; second the arcs $\left(D_{k}, T_{i}\right)$ and $\left(T_{i}, D_{k}\right)$, for every $k$ in $\{1,2, \ldots,|K|\}$ and every $i$ in $\{1,2, \ldots, n\}$.

To simplify the notation, we will assign the index $n+k$ to the vertex $D_{k}$ (for every $k$ ) and use the notation $(i, j, k)$ for an $\operatorname{arc}$ of $G$. Thus if $i$ and $j$ are at most $n$, $(i, j, k)$ refers to the $\operatorname{arc}\left(T_{i}, T_{j}, k\right)$. If $i$ (resp. $\left.j\right)$ is greater than $n$, then $i$ (resp. $j$ ) equals $n+k$ and $(n+k, j, k)$ [resp. $(i, n+k, k)]$ refers to an arc of the form $\left(D_{k}, T_{j}\right)$ [resp. $\left(T_{i}, D_{k}\right)$ ]. There are several copies of the "couple" $(i, j)$ (for $\left.i, j \leq n\right)$ because if such an arc appears in a feasible solution of the MDVSP instance, it must belong to a route starting and ending with some depot $D_{k}$. In the present article, as in Hadjar et al. (2006), we shall identify depots with colors, that is, we will refer to an index in $\{1,2, \ldots,|K|\}$ as a "color". Note also that the subgraph induced by $\left\{T_{1}, T_{2}, \ldots, T_{n}\right\}$ is acyclic, since the arcs of this subgraph describe a precedence relation between the trips. Clearly there is a one-to-one correspondence between routes (as defined in the previous paragraph) and monochromatic circuits containing exactly one depot. A feasible assignment is a partition of the set of trips into monochromatic circuits in $G$ such that the number of circuits including $D_{k}$ is at most $v_{k}$ (for any $k$ ).

We now define the cost of such a partition. The cost of arc $(i, j, k)$ is denoted by $c_{i j}$ for all $i, j$, and $k$ (observe that if $c_{i j}$ were replaced by $c_{i j}^{k}$ and thus depended upon $k$, there would be no substantial change in the rest of the present article). Let $R$ denote a route, i.e., a sequence of the form $\left(n+k, i_{1}, \ldots, i_{\ell}, n+k\right)$ for some depot $D_{k}$ and some trips $T_{i_{1}}, \ldots, T_{i_{\ell}}$, where $\ell$ is at least 1 . We will also consider $R$ as a set of arcs of color $k$, i.e., as the set $\left\{\left(D_{k}, T_{i_{1}}\right),\left(T_{i_{1}}, T_{i_{2}}\right), \ldots,\left(T_{i_{\ell}}, D_{k}\right)\right\}$. Then the cost of $R$ is defined as $\sum_{(i, j, k) \in R} c_{i j}$. The cost of a feasible assignment is the sum of the costs of all the routes contained in it. We define the MDVSP as follows: given a multigraph $G$, availabilities $v_{k}$ for the depots, and costs $c_{i j}$ for the arcs in $G$, find a 
feasible assignment of minimum cost. Recall that the availability $v_{k}$ is the maximum number of vehicles that can be housed in the $k$ th depot.

To formulate the MDVSP as an integer program, we introduce a binary variable $X_{i j}^{k}$ for every arc $(i, j, k)$ in $A$ and define it as follows: $X_{i j}^{k}$ equals 1 if $(i, j, k)$ belongs to a route in the feasible assignment and 0 otherwise. Also we let $\sigma^{+}(i)$ denote the set of "successors" of $i$ (i.e., the set $\{j \mid(i, j, k) \in A$ for some $k\})$ and $\sigma^{-}(i)$ the set of predecessors of $i$ (i.e., the set $\{j \mid(j, i, k) \in A$ for some $k\})$. The multicommodity flow model for the MDVSP is the following integer program, denoted by $(P)$ (see Desrosiers et al. 1995).

$$
\min \sum_{(i, j, k) \in A} c_{i j} X_{i j}^{k}
$$

subject to

$$
\begin{array}{cl}
\sum_{k \in K} \sum_{j \in \sigma^{+}(i)} X_{i j}^{k}=1 \quad & \forall i \in\{1,2, \ldots, n\} \\
\sum_{j=1}^{n} X_{n+k, j}^{k} \leq v_{k} & \forall k \in K \\
\sum_{j \in \sigma^{-}(i)} X_{j i}^{k}-\sum_{j \in \sigma^{+}(i)} X_{i j}^{k}=0 \quad \forall k \in K, \quad \forall i \in\{1,2, \ldots, n, n+k\} \\
X_{i j}^{k} \in\{0,1\} \quad \forall(i, j, k) \in A
\end{array}
$$

Note that in any feasible solution of $(P)$,

- the set of arcs of color $k$ (for any $k$ ) can be partitioned into circuits of colour $k$, i.e., circuits containing depot $D_{k}$ [because of Constraints (3)],

- the number of circuits of color $k$ is at most $v_{k}$, the availability at $D_{k}$ [because of Constraints (2)], and

- the collection of all such circuits is actually a partition of the set of trips [because of Constraints (1)].

The goal of the model is to minimize the total cost of a feasible assignment, i.e., the objective function $\sum_{(i, j, k) \in A} c_{i j} X_{i j}^{k}$.

It is well known that any feasible solution vector of the multicommodity flow model (or its linear programming relaxation) can be expressed as a weighted sum of vectors such that

- each vector is the incidence vector of a monochromatic circuit,

- each weight is positive, and

- the sum of the weights equals the value of the feasible solution.

We shall refer to the weight of the incidence vector of circuit $p$ as the weight of $p$ and denote it by $w_{p}$. We also denote by $\mathcal{P}$ the set of all circuits appearing in such a decomposition of a particular feasible solution vector; thus $X_{i j}^{k}>0$ holds if the arc $(i, j, k)$ belongs to a circuit $p \in \mathcal{P}$. 
Consider now any feasible solution of the linear programming relaxation of $(P)$, with the $w_{p}$ and $\mathcal{P}$ defined as in the previous paragraph. Such a solution often contains a conflictual structure of the form $\left(S_{1}, \mathcal{P}^{\prime}\right)$, where

- $\mathcal{P}^{\prime}$ is a subset of $\mathcal{P}$,

- $S_{1}$ is an odd set of trips,

- each circuit in $\mathcal{P}^{\prime}$ contains exactly two trips belonging to $S_{1}$.

For each circuit $p$ in $\mathcal{P}^{\prime}$ we denote by $\hat{p}$ the subpath of $p$ between the two trips in $S_{1}$ that belong to $p$. Note that the subscript 1 in $S_{1}$ does not refer to the subpaths length but to the fact that the conflictual structure also contains intermediate trips or vertices; we refer the reader to Definition 1 below. The weight of $\hat{p}$ is equal to that of $p$. As an example, consider the middle graph (which is a directed graph) in Fig. 1. In this example, there are two depots (or colors), $D_{1}$ and $D_{2}$, and the set $S_{1}$ includes the vertices $a, b$ and $c$. The color 1 is represented in boldface type to distinguish it from the color 2 . The paths in $\mathcal{P}^{\prime}$ are $\left(D_{1}, a, b, D_{1}\right),\left(D_{2}, b, c, D_{2}\right)$, and $\left(D_{2}, a, d, c, D_{2}\right)$. The vertex $d$ is an intermediate vertex; hence it does not belong to $S_{1}$. The weight of each path in $\mathcal{P}^{\prime}$ equals 0.5 and the arc $(e, d)$ (of color 1 ) belongs to a path that is not a member of $\mathcal{P}^{\prime}$.

In general, given a conflictual structure $\left(S_{1}, \mathcal{P}^{\prime}\right)$, Constraints (1) and (3) imply that for any $u$ in $S_{1}$, the sum of the weights of the subpaths having $u$ as an endpoint is at most 1 . Hence if we define an undirected graph with vertex set $S_{1}$ and edge set $E=\{u v \mid u$ and $v$ are the endpoints of $\hat{p}$ for some $p\}$ and we assign to edge $u v$ the weight of the corresponding subpath, then the vector of edge weights in the undirected graph $\left(S_{1}, E\right)$ is actually a solution of the linear relaxation of the matching problem for the graph $\left(S_{1}, E\right)$. In Fig. 1 the graph on the left is the graph $\left(S_{1}, E\right)$ obtained from the middle graph by replacing each subpath by an edge. Conversely, given a blossom in an undirected graph, one can obtain a conflictual structure by replacing each blossom edge by a directed path, as illustrated in Fig. 1. The phrase "conflictual structure" refers to the fact that there is a conflict at each vertex in $S_{1}$. In our example, there is a conflict at vertex $a$ because subpaths $(a, b)$ and $(a, d, c)$ have different colors, a conflict at vertex $b$ because $(a, b)$ and $(b, c)$ have different colors, and a conflict at vertex $c$ because $c$ is the head of subpaths $(b, c)$ and $(a, d, c)$.

Given the relationship just described between some fractional solutions of $(P)$ and those of the matching problem, we should expect a similar relationship between certain cutting planes for $(P)$ and the cutting planes based on blossoms for the matching problem formulation. For more information on the facets of the matching polytope, we refer the reader to Pulleyblank and Edmonds (1974) and Nemhauser and Wolsey (1988). As shown in Hadjar et al. (2006), the description of a valid inequality "cutting" the fractional solution displayed in the middle graph of Fig. 1 requires the introduction of another arc (a thorn), which is incident upon the vertex $d$. In our example this thorn is the arc $(e, d)$. Finally, Fig. 1 contains a description of the conflict graph of the partial MDVSP solution, in which two vertices (corresponding to arcs in the middle graph) are joined by an edge if and only if they are in conflict. The valid inequality corresponding to this conflictual structure is $X_{a b}^{1}+X_{b c}^{2}+X_{a d}^{2}+X_{d c}^{2}+X_{e d}^{1} \leq 2$.

The many examples of conflictual structures in fractional solutions of the MDVSP led Hadjar et al. (2006) to introduce thorny submultigraphs (see Definition 4.4 in their 

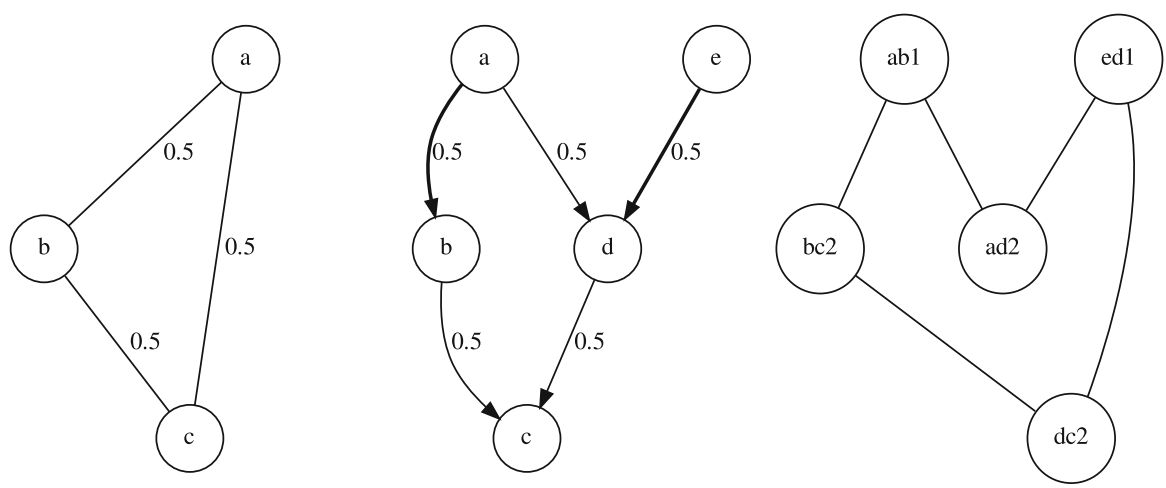

Fig. 1 A blossom, a conflictual structure obtained by replacing each edge of the blossom by a subpath, and the conflict graph corresponding to the structure. Note that a vertex of the conflict graph corresponds to an arc of the middle graph and is denoted by $u v k$, where $u$ is the tail of the arc, $v$ the head of the arc, and $k$ its color

article). These submultigraphs do not necessarily look like cycles, as demonstrated in Fig. 2, which includes a vertex in $S_{1}$ (the vertex $T 204$ ) that is of degree 1 . The definition given in Hadjar et al. (2006) can be broadened slightly and we give the "broad" definition below. Note that for any subsets $A^{\prime}$ and $F$ of $\operatorname{arcs}$ in $G, \operatorname{col}\left(A^{\prime}\right)$ denotes the set of colors of $\operatorname{arcs}$ in $A^{\prime}$ and $\delta_{F}^{+}(i)$ [resp. $\left.\delta_{F}^{-}(i)\right]$ the set of arcs belonging to $F$ whose tail (resp. head) is $i$. When $F$ is the set of all arcs, we omit $F$ and use the notations $\delta^{+}(i)$ and $\delta^{-}(i)$. Definition 1 is illustrated in Fig. 2.

Definition 1 Let $H=(S, F)$ be a submultigraph of $G$. We say that $H$ is a thorny submultigraph if $S$ can be partitioned into sets $S_{1}, S_{2}$, and $S_{3}$ and $F$ can be partitioned into sets $F_{1}$ and $F_{2}$ verifying the properties listed below. The arc set $F_{2}$ is itself the disjoint union of the family $\{\tau(v)\}_{v \in S_{2}}$, where $\tau(v)$ (the set of thorns associated with the vertex $v$ ) is a subset of arcs incident upon $v$.

1. $S_{1} \cup S_{2}$ does not contain any depot.

2. $\left|S_{1}\right|$ is odd and at least 3 .

3. $\operatorname{col}\left(\delta_{F_{1}}^{-}(v)\right) \cap \operatorname{col}\left(\delta_{F_{1}}^{+}(v)\right)$ is empty for any $v$ in $S_{1}$.

4. For any $v$ in $S_{2}$, the arc set $\tau(v)$ is either a subset of $\delta^{+}(v)$ or a subset of $\delta^{-}(v)$.

5. $\operatorname{col}(\tau(v)) \cap \operatorname{col}\left(\delta_{F_{1}}^{-}(v)\right)$ and $\operatorname{col}(\tau(v)) \cap \operatorname{col}\left(\delta_{F_{1}}^{+}(v)\right)$ are both empty for any $v$ in $S_{2}$.

6. Any arc incident to a vertex in $S_{3}$ belongs to $\tau(v)$ for some $v$ in $S_{2}$.

Note that for a given submultigraph $H=(S, F)$, there might be more than one way of partitioning $S$ into $S_{1}, S_{2}$, and $S_{3}$ and $F$ into $F_{1}$ and $F_{2}$. In general, however, if we consider a specific solution of the linear relaxation of $(P)$, at most one partition will yield a violated inequality.

There are two differences between the above definition and that found in Hadjar et al. (2006). The first difference is that we have removed the requirement that $\delta_{F_{2}}^{-}(v)$ be empty for $v$ in $S_{1}$ (which used to appear in Property no. 3); this requirement is not used in the proof that Inequalities (4) are valid (see below). The second difference is 


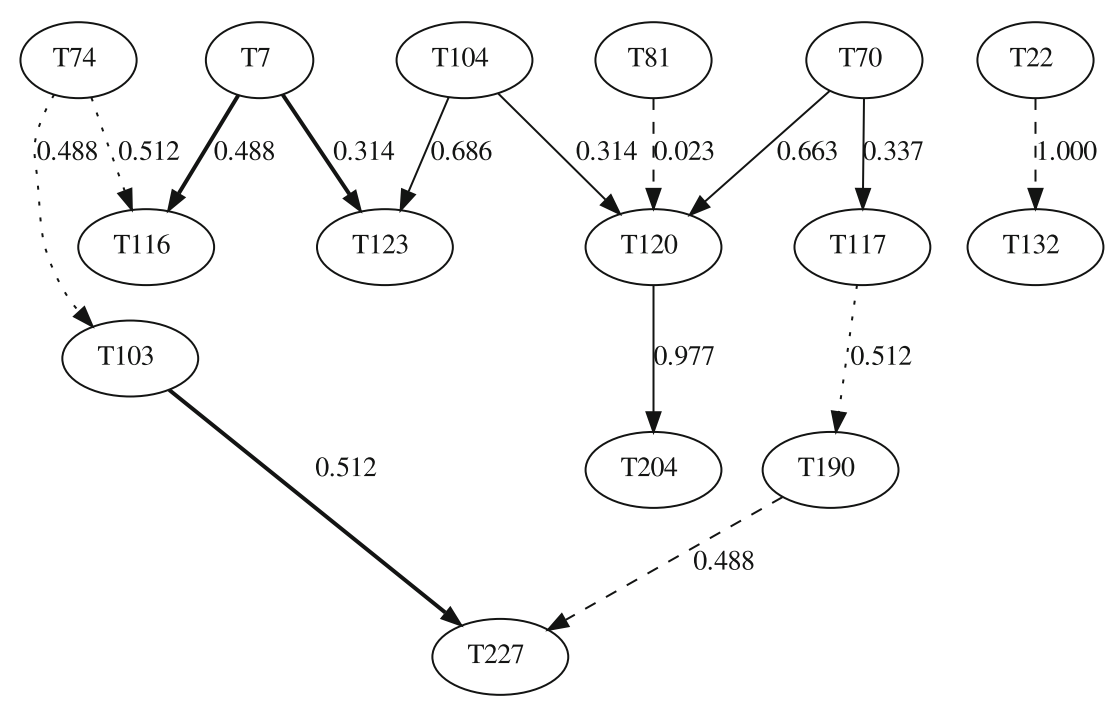

Fig. 2 A thorny submultigraph. Only the arcs with a nonzero flow have been included into the figure. There are four colors, represented by boldface arcs, light arcs, dashed arcs, and dotted arcs (respectively). All the nodes belong to $S_{1}$, except for $T 120$, which belongs to $S_{2}$, and $T 81$, which belongs to $S_{3}$. All the arcs belong to $F_{1}$, except for the thorn $(T 81, T 120)$, which belongs to $F_{2}$. Note that there is a conflict at every node in $S_{1}$; for instance $T 74$ is the tail of both $(T 74, T 103)$ and $(T 74, T 116)$ and the colors of the two arcs incident upon $T 103$ are different. Although $(T 104, T 120)$ is not in conflict with $(T 120, T 204)$, the thorn ( $T 81, T 120)$ is in conflict with the three other arcs incident upon $T 120$. Adding up all the flow values of the arcs in $F_{1} \cup F_{2}$ demonstrates that Inequality (4) is violated

that in the above definition, $\tau(v)$ (the set of thorns) is not always a subset of $\delta_{F_{2}}^{-}(v)$; it may also be a subset of $\delta_{F_{2}}^{+}(v)$. Thus either all the thorns in $\tau(v)$ have $v$ as their head or all of them have $v$ as their tail.

Proposition 1 Let $H=(S, F)$ be a thorny submultigraph of $G$, where $S=S_{1} \cup S_{2} \cup S_{3}$ and $F=F_{1} \cup F_{2}$. The inequality

$$
\sum_{(i, j, k) \in F} X_{i j}^{k} \leq\left\lfloor\left|S_{1}\right| / 2\right\rfloor+\left|S_{2}\right|
$$

is valid for $(P)$.

Proof We first show that for any $i \in\{1,2, \ldots, n\}$ and any subsets $K_{1}$ and $K_{2}$ of $K$ verifying $K_{1} \cap K_{2}=\emptyset$, the following inequality holds.

$$
\sum_{k \in K_{1}} \sum_{j \in \sigma^{+}(i)} X_{i j}^{k}+\sum_{k \in K_{2}} \sum_{j \in \sigma^{-}(i)} X_{j i}^{k} \leq 1
$$

First observe that for any $i \in\{1,2, \ldots, n\}$, Inequality (1) implies that

$$
\sum_{k \in K_{1}} \sum_{j \in \sigma^{+}(i)} X_{i j}^{k}+\sum_{k \in K_{2}} \sum_{j \in \sigma^{+}(i)} X_{i j}^{k} \leq 1
$$


holds whenever the intersection of $K_{1}$ and $K_{2}$ is empty. From the Inequalities (3) we deduce that

$$
\sum_{j \in \sigma^{+}(i)} X_{i j}^{k}=\sum_{j \in \sigma^{-}(i)} X_{j i}^{k}
$$

holds for any $i \in\{1,2, \ldots, n\}$ and any $k \in K$ and thus

$$
\sum_{k \in K_{1}} \sum_{j \in \sigma^{+}(i)} X_{i j}^{k}+\sum_{k \in K_{2}} \sum_{j \in \sigma^{-}(i)} X_{j i}^{k} \leq 1
$$

holds as well.

Assume now that $v$ is any vertex and the subsets of $\operatorname{arcs} A_{1}$ and $A_{2}$ verify the conditions that $A_{1}$ (resp. $A_{2}$ ) is a subset of $\delta^{+}(v)$ or a subset of $\delta^{-}(v)$ and the intersection of $\operatorname{col}\left(A_{1}\right)$ and $\operatorname{col}\left(A_{2}\right)$ is empty. Under those conditions, Inequalities (1) and (5) imply the following inequality.

$$
\sum_{(i, j, k) \in A_{1} \cup A_{2}} X_{i j}^{k} \leq 1
$$

From Inequalities of the form (6) we can deduce Inequalities (4) by applying the Chvátal-Gomory procedure. Indeed consider the following inequalities.

$$
\begin{gathered}
\sum_{(i, j, k) \in \delta_{F_{1}}^{-}(v) \cup \delta_{F_{1}}^{+}(v)} X_{i j}^{k} \leq 1 \text { for any } v \in S_{1} \\
\sum_{(i, j, k) \in \tau(v) \cup \delta_{F_{1}}^{-}(v)} X_{i j}^{k} \leq 1 \text { for any } v \in S_{2} \\
\sum_{(i, j, k) \in \tau(v) \cup \delta_{F_{1}}^{+}(v)} X_{i j}^{k} \leq 1 \text { for any } v \in S_{2}
\end{gathered}
$$

The validity of each inequality within those three groups follows from the validity of Inequalities (6) and the definition of thorny submultigraph.

A variable $X_{i j}^{k}$ corresponding to an arc $(i, j, k)$ in $F_{1}$ appears exactly twice in this family of inequalities, since both its tail and its head belong to $S_{1} \cup S_{2}$. Also a variable $X_{i j}^{k}$ corresponding to an arc $(i, j, k)$ in $F_{2}$ appears exactly twice in this family of inequalities. Multiplying each inequality by $1 / 2$ yields

$$
\sum_{(i, j, k) \in F} X_{i j}^{k} \leq \frac{\left|S_{1}\right|+2\left|S_{2}\right|}{2}
$$

and therefore

$$
\sum_{(i, j, k) \in F} X_{i j}^{k} \leq\left\lfloor\left|S_{1}\right| / 2\right\rfloor+\left|S_{2}\right| .
$$


In the terminology of Caprara and Fischetti (1996), Inequalities (4) are $\{0,1 / 2\}$ Chvátal-Gomory cuts, as are the odd-cycle and blossom inequalities in matching theory.

Hadjar et al. (2006) proved that under some assumptions, Inequalities (4) (the socalled odd-set inequalities) define facets of the convex hull of integral solutions of model $(P)$ (note that Löbel 1997 had already shown that some trivial inequalities define facets). Separating Inequalities (4) (i.e., finding violated inequalities of this type) seems to be a difficult problem and we make the following conjecture.

Conjecture 1 The problem of separating Inequalities (4) is NP-hard.

In "Finding violated valid inequalities through a search of the conflict graph" we present a method for separating a subclass of such inequalities, which we call the oddcycle inequalities. In "Finding violated valid inequalities through an auxiliary integer program" we show how to separate a much broader subclass of Inequalities (4).

\section{Finding violated valid inequalities through a search of the conflict graph}

Hadjar et al. (2006) present a strategy based on enumeration to find some valid inequalities of type (4) that are violated by the optimal solution of the linear relaxation of $(P)$. Let $G=(V, A)$ be the multigraph underlying an MDVSP instance. We say that the two $\operatorname{arcs}(i, j, k)$ and $\left(i^{\prime}, j^{\prime}, k^{\prime}\right)$ of $G$ are in conflict if $i=i^{\prime}$ holds and $i$ is not a depot or $j=j^{\prime}$ holds and $j$ is not a depot or they have a vertex in common and different colors. A thorny cycle is a sequence of arcs in $G$ starting and ending with the same arc and such that each arc in the sequence is in conflict with the arcs preceding and following it. We shall explain below the relationship between thorny submultigraphs and thorny cycles containing an odd number of arcs. Figure 3 illustrates a thorny cycle. The

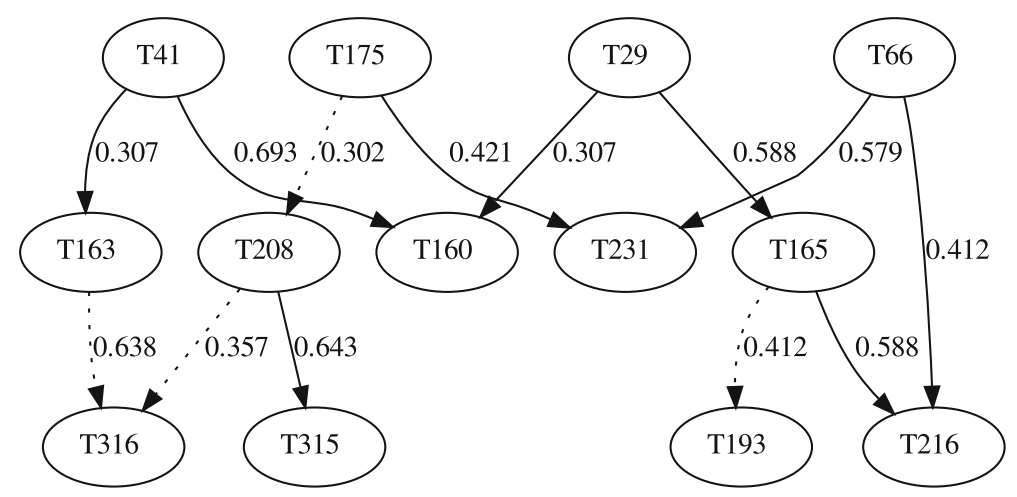

Fig. 3 A thorny cycle (with two arc colors). The following is the arc sequence: $(T 41, T 163,1)$, (T163, T316, 2), (T208, T316, 2), (T208, T315, 1), (T175, T208, 2), (T175, T231, 1), (T66, T231, 1), (T66, T216, 1), (T165, T216, 1), (T165, T193, 2), (T29, T165, 1), (T29, T160, 1), (T41, T160, 1). This thorny cycle is also a thorny submultigraph, in which all the nodes belong to $S_{1}$, except for $T 165$ and $T 208$, which belong to $S_{2}$, and $T 193$ and $T 315$, which belong to $S_{3}$. Adding up all the arc flow values demonstrates that Inequality (4) is violated 
procedure in Hadjar et al. (2006) looks for violated valid inequalities corresponding to some thorny cycles. It has two drawbacks:

- enumeration of the cycles is time consuming, even if one is only looking for cycles of bounded length; and

- the procedure cannot detect more general structures giving rise to violated inequalities.

Furthermore, Definition 1 is slightly broader than that used in Hadjar et al. (2006). In this section, we propose an algorithm that is more efficient than the previous one.

We introduce the (undirected) conflict graph of $G$, denoted $L(G)$. The vertex set of $L(G)$ is $A$ and there is an edge between the vertices $(i, j, k)$ and $\left(i^{\prime}, j^{\prime}, k^{\prime}\right)$ if and only if these two arcs are in conflict. Clearly, two $\operatorname{arcs}(i, j, k)$ and $\left(i^{\prime}, j^{\prime}, k^{\prime}\right)$ cannot both belong to a solution if they are in conflict. Therefore, any integral solution of the MDVSP instance corresponds to a stable set in $L(G)$. The converse is not always true because a solution of the MDVSP must satisfy the covering and availability constraints. Similarly, any thorny cycle in $G$ gives rise to a cycle in $L(G)$. Also, any fractional solution of the MDVSP instance gives rise to a fractional stable set in $L(G)$ (recall that a fractional stable set in an undirected graph $H$ is an assignment of weights $x_{i}$ to the vertices of $H$ such that $x_{i}+x_{j} \leq 1$ holds for every edge $i j$ in $H$ ). This observation can be used to find, in polynomial time, violated inequalities based on thorny cycles. Let $G^{\prime}=\left(V, A^{\prime}\right)$ denote the support of the optimal solution of the linear relaxation of $(P)$, i.e., the submultigraph of $G$ defined by

$$
A^{\prime}=\left\{(i, j, k) \mid X_{i j}^{k}>0\right\}
$$

If two vertices $(i, j, k)$ and $\left(i^{\prime}, j^{\prime}, k^{\prime}\right)$ of $L\left(G^{\prime}\right)$ are joined by an edge $e$, we assign the weight $w_{e}=1-X_{i j}^{k}-X_{i^{\prime} j^{\prime}}^{k^{\prime}}$ to this edge (where $X_{i j}^{k}$ denotes the value of the flow on $\operatorname{arc}(i, j, k)$ in the optimal solution of the linear relaxation of $(P))$.

We claim that to find a violated odd cycle inequality for the stable set polytope of $L\left(G^{\prime}\right)$, it suffices to find an odd cycle of weight less than 1 in $L\left(G^{\prime}\right)$ (with the edge weights that we have just introduced). As in Nemhauser and Sigismondi (1992), we define the weight of a cycle $C$ in $L\left(G^{\prime}\right)$ (denoted by $w(C)$ ) as $\sum_{e \in C} w_{e}$ and assume that there is an odd cycle $C$ with $w(C)<1$, i.e.,

$$
\sum_{e \in C}\left(1-X_{i j}^{k}-X_{i^{\prime} j^{\prime}}^{k^{\prime}}\right)<1,
$$

where $e$ denotes the edge joining $(i, j, k)$ to $\left(i^{\prime}, j^{\prime}, k^{\prime}\right)$. This inequality is equivalent to

$$
|C|-2\left(\sum_{(i, j, k) \in C} X_{i j}^{k}\right)<1
$$


and thus to

$$
\frac{|C|-1}{2}<\sum_{(i, j, k) \in C} X_{i j}^{k}
$$

We conclude that $C$ gives rise to a violated odd cycle inequality for the stable set polytope corresponding to $L\left(G^{\prime}\right)$ (or $L(G)$ ). Actually, the strongest inequalities in this family are those corresponding to chordless cycles since the cycles containing chords do not induce facets of the stable set polytope. We now show how to use these observations in the context of the model $(P)$ and valid inequalities of type (4).

We start by showing that a chordless odd cycle $C$ in $L\left(G^{\prime}\right)$ or $L(G)$ (arising from a thorny cycle in $G$ ) actually corresponds to a thorny submultigraph of $G$. We assume that $C$ is of length greater than 3 (i.e., at least equal to 5 ), denote by $a_{0}, a_{1}, \ldots, a_{s-1}$ the vertices in $C$, and use the same notation for the arcs of $G$ corresponding to these vertices. Similarly, we use $C$ to denote the sequence of $\operatorname{arcs} a_{0}, a_{1}, \ldots, a_{s-1}$ in $G$. We denote by $V_{t}$ (for $t=0,1, \ldots, s-1$ ) the set of vertices of $G$ incident to $a_{t}$ (i.e., if $a_{t}$ is the arc $(i, j, k)$, then $V_{t}$ is the set $\left.\{i, j\}\right)$. We observe that

- any $i$ in $\bigcup_{t=0}^{s-1} V_{t}$ is incident to at most four arcs in $C$, and

- if $i$ is incident to four arcs, then these arcs have the same color and two of the arcs incident upon $i$ are incoming arcs while the other two are outgoing arcs.

The first statement follows because if $i$ were incident to at least five arcs, $i$ would be the head of three arcs in $C$ or the tail of three arcs in $C$. Then these three arcs would form a triangle in $L(G)$, contradicting the assumption that $C$ is of length greater than 3 and chordless. For exactly the same reason, if $i$ is incident to four arcs only, then two of the arcs are incoming and two are outgoing arcs. Finally, if not all four arcs have the same color, it is easily shown that $C$ includes a triangle or a 4-cycle, contradicting the assumption that $C$ is chordless.

Figure 4 illustrates the situation where a vertex of $G$ is incident to four arcs corresponding to vertices in the chordless cycle $C$. Note that in Fig. 4, the only vertex incident to four arcs in $C$ (namely the vertex $T 294$ ) is a cut vertex. That is not always the case since the submultigraph of $G$ consisting of all the arcs in $C$ may be 2-connected (if it includes several vertices incident to four arcs in $C$ ). This possibility is illustrated in Fig. 5.

To show that the cycle $C$ corresponds to a thorny submultigraph, we have to give an explicit description of the sets $S_{1}, S_{2}, S_{3}, F_{1}$, and $F_{2}$. We use Algorithm 1 below to build these five sets. Note that in an expression such as $V_{t+1}$ or $V_{t+2}$, the subscript must be taken modulo $s$. The algorithm proceeds by assigning each $\operatorname{arc} a_{t}$ to $F_{1}$ or $F_{2}$, in the order $a_{0}, a_{1}, \ldots, a_{s-1}$. However, we must choose $a_{0}$ carefully. The following three cases exhaust all possibilities.

1. If every vertex in $\bigcup_{t=0}^{s-1} V_{t}$ is incident to at most two $\operatorname{arcs}$ in $C$, then we choose as $a_{0}$ any arc in $C$.

2. If some vertex $i$ in $\bigcup_{t=0}^{s-1} V_{t}$ is incident to exactly four arcs in $C$, then we choose as $a_{0}$ one of the arcs incident upon $i$.

3. Assume that no vertex in $\bigcup_{t=0}^{s-1} V_{t}$ is incident to exactly four arcs, but some vertex $i$ is incident to exactly three $\operatorname{arcs}$ (say, $a, a^{\prime}$, and $a^{\prime \prime}$ ). Because $C$ is chordless, at 


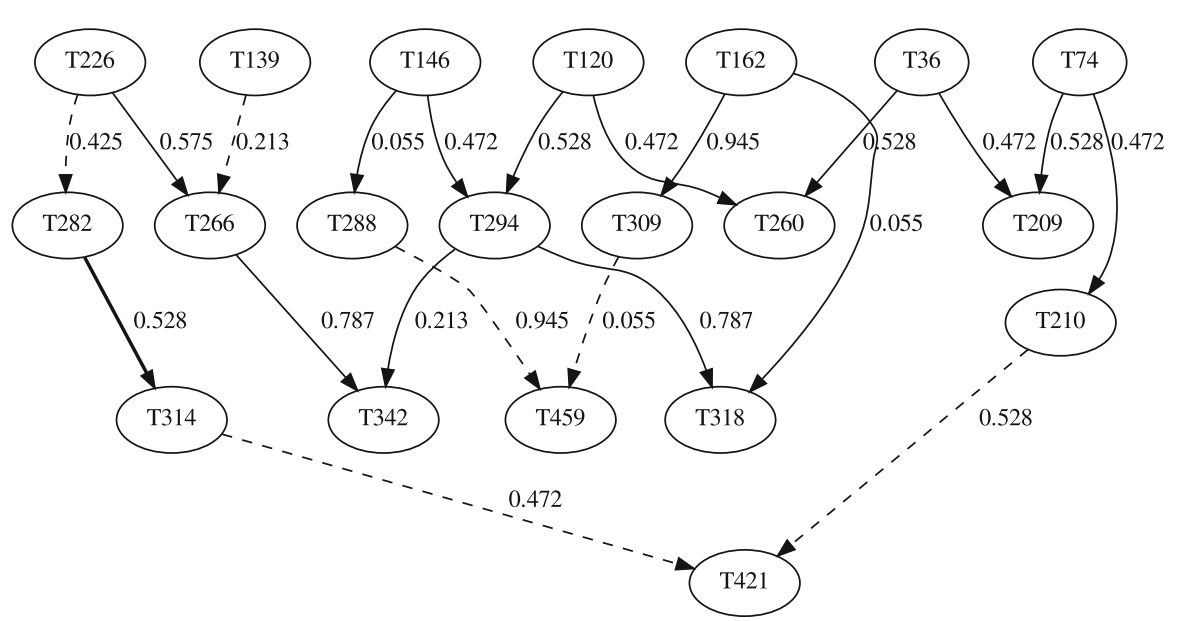

Fig. 4 A thorny submultigraph. Only the arcs with a nonzero flow have been included in the figure. All the nodes belong to $S_{1}$, except for $T 266$ and $T 294$, which belong to $S_{2}$, and $T 139$, which belongs to $S_{3}$. Note that $T 294$ is a cut vertex. Adding up all the flow values demonstrates that inequality (4) is violated

Fig. 5 A thorny submultigraph in which two vertices are of degree 4 and all the others of degree 2 . There are two colors in this example: one represented by boldface lines and the other by light lines. The corresponding structure in the conflict graph is a chordless cycle on 13 vertices. This is easily verified by scanning the vertices in the following order: $0,1,2,3,4,5$, $6,7,2,8,9,5,10,0$

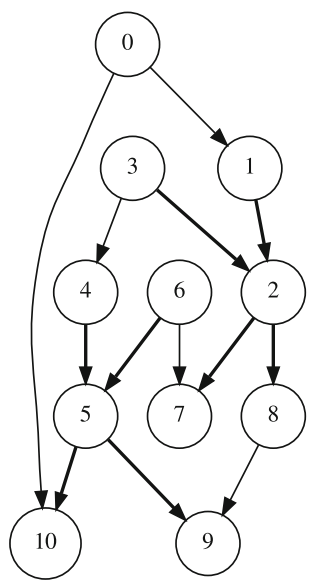

least two of these arcs are not in conflict. If the subgraph induced by $a, a^{\prime}$, and $a^{\prime \prime}$ in $L\left(G^{\prime}\right)$ contains two edges, then there must be an index $t$ such that $i$ is incident to $a_{t}, a_{t+1}$, and $a_{t+2}$. Then we choose as $a_{0}$ the $\operatorname{arc} a_{t}$, i.e., we assign labels to the arcs in such a way that $a_{t}$ is the first arc in the sequence. If the subgraph induced by $a, a^{\prime}$, and $a^{\prime \prime}$ in $L\left(G^{\prime}\right)$ contains one edge only, then there must be an index $t$ such that $i$ is incident to $a_{t}$ and $a_{t+1}$. Then we choose as $a_{0}$ the arc $a_{t}$.

It is straightforward to verify that the five sets $F_{1}, F_{2}, S_{1}, S_{2}$, and $S_{3}$ constructed by Algorithm 1 verify the conditions of Definition 1 . The odd cycle inequality corresponding to $C$ in the stable set polytope of $L(G)$ (i.e., $\sum_{e \in C} w_{e} \geq 1$ or $\left.\left.\sum_{(i, j, k) \in C} X_{i j}^{k} \leq|C|-1\right) / 2\right)$ is precisely the inequality corresponding to the thorny submultigraph that we have just constructed, i.e., $\sum_{(i, j, k) \in F} X_{i j}^{k} \leq\left\lfloor\left|S_{1}\right| / 2\right\rfloor+\left|S_{2}\right|$. 


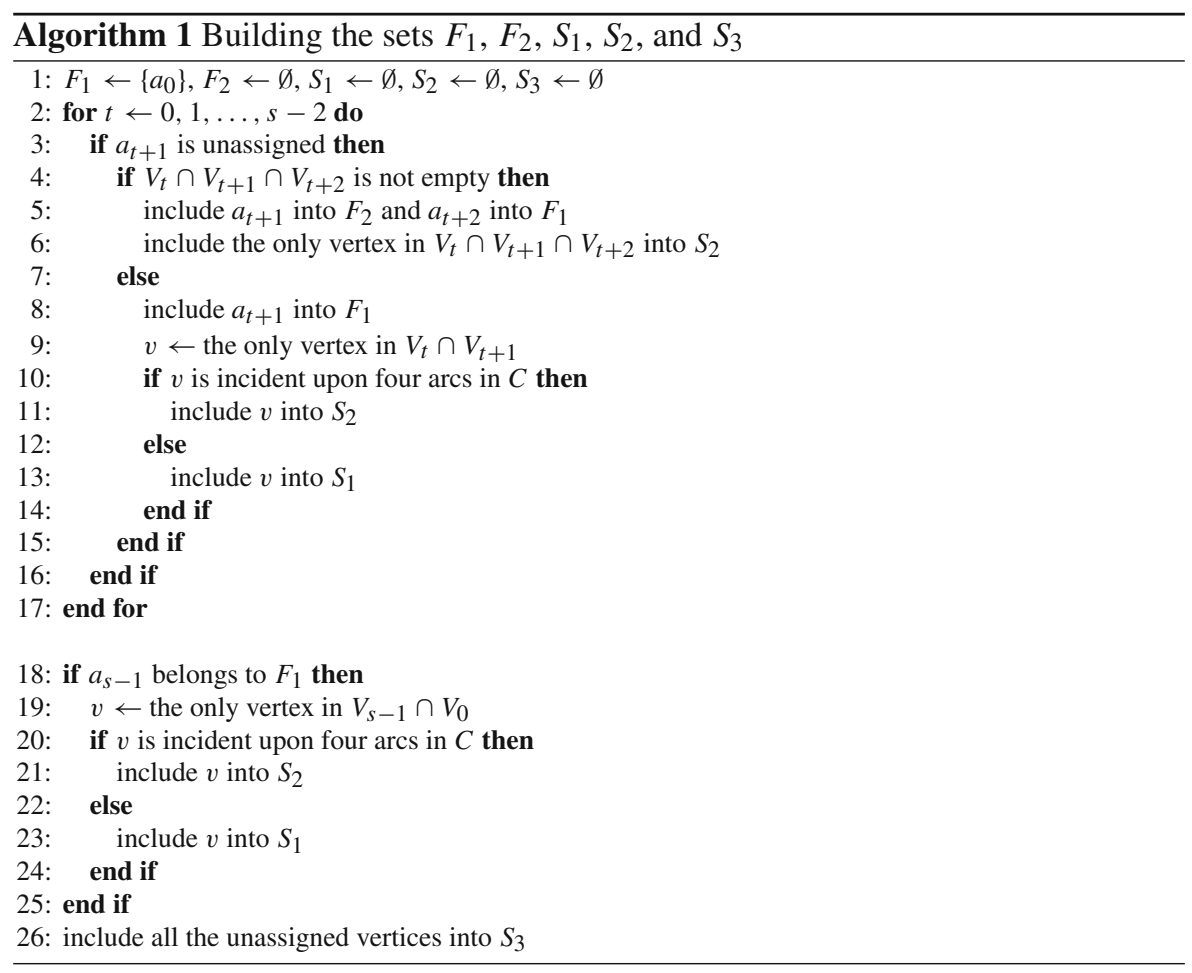

Recall that we have assumed that $C$ was of length greater than 3 . The case where the length of $C$ equals 3 is straightforward and we leave it to the reader.

We now consider the problem of finding those thorny cycles in $G$ giving rise to chordless cycles in $L\left(G^{\prime}\right)$. As in Nemhauser and Sigismondi (1992), we create a bipartite graph $B$ with two copies of each vertex and two copies of each edge of $L\left(G^{\prime}\right)$. To find an odd cycle of smallest weight containing vertex $(i, j, k)$, we compute a shortest path from one copy of $(i, j, k)$ to the other in the bipartite graph $B$. The cycle returned by this procedure may contain chords and may not even be minimal (when considered as a set of vertices). For the cycle to be as small as possible (and thus yield an inequality that is as strong as possible), we add a small quantity (denoted by $\epsilon$ ) to each weight $w_{e}$ in $L\left(G^{\prime}\right)$ and $B$ (this method is used for instance in Section 5.2.1 of Rebennack 2006). If we compute a minimum-weight odd cycle (denoted by $C$ ) containing the vertex $(i, j, k)$ and having total weight less than 1 , then only two cases may arise:

- $C$ does not contain any chord and can be included in the list of cycles yielding violated valid inequalities; and

- $C$ does contain chords, but none of these chords defines an odd cycle containing vertex $(i, j, k)$.

In the second case, we include into the list of cycles yielding violated valid inequalities all minimal odd cycles defined by chords (where "minimal" means "minimal with 
respect to set inclusion"). It is easily verified that for each such cycle $C^{\prime}$, the inequality $\sum_{e \in C^{\prime}} w_{e}<1$ is verified. Algorithm 2 summarizes our procedure.

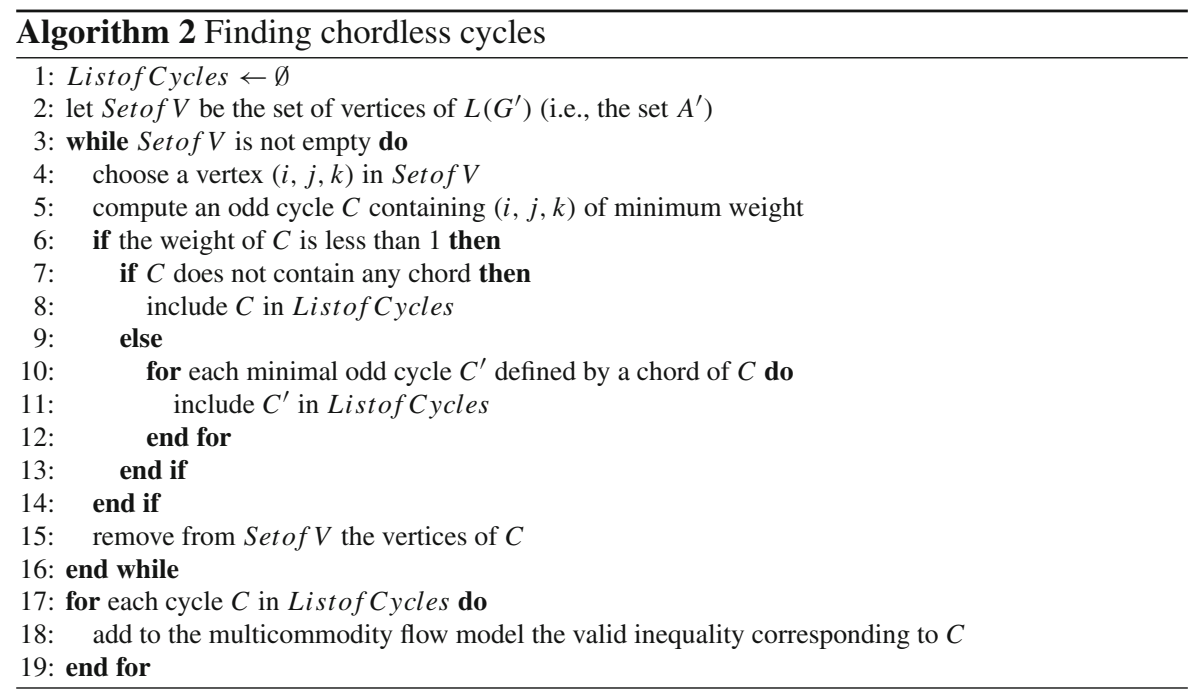

Recall that the valid inequality corresponding to cycle $C$ is $\sum_{(i, j, k) \in C} X_{i j}^{k} \leq$ $(|C|-1) / 2$. It is possible to strengthen this inequality by lifting it, i.e., by including other variables in its left-hand side. We now describe a lifting procedure for cycle $C$. Let us denote the arcs in $C$ by $a_{0}, a_{1}, \ldots, a_{2 t}$, where $t$ is at least 1 and the arcs $a_{\ell-1}$ and $a_{\ell}$ are in conflict for every $\ell$ comprised between 0 and $2 t$ (where $\ell-1$ is taken modulo $2 t+1)$. Let us also denote by $Q_{\ell}$ the set $\left\{a_{\ell-1}, a_{\ell}\right\}$ and by $S_{\ell}$ the set of vertices common to $a_{\ell-1}$ and $a_{\ell}$. Note that $Q_{\ell}$ is a clique in $L(G)$ and that $S_{\ell}$ cannot be empty because $a_{\ell-1}$ and $a_{\ell}$ are in conflict. Finally, let $a=(u, v, k)$ denote an arc that is not in $C$ and not incident to any depot. If there exist two indices $\ell$ and $\ell^{\prime}$ in $\{0,1, \ldots, 2 t\}$ such that $S_{\ell} \cap\{u, v\}$ and $S\left(\ell^{\prime}\right) \cap\{u, v\}$ are nonempty and $Q_{\ell} \cup\{a\}$ and $Q_{\ell^{\prime}} \cup\{a\}$ are both cliques in $L(G)$, then the variable $X_{u v}^{k}$ can be included into the left-hand side of the valid inequality corresponding to $C$. Algorithm 3 summarizes our procedure, where $C^{\prime}$ denotes the "enriched" cycle.

Note that this lifting is carried out with arcs in $G^{\prime}$, i.e., arcs that have a positive flow in the linear programming relaxation. After this lifting we apply the same procedure but use the zero-flow arcs instead. In practice, we have noticed that few arcs were added to the cycle during the lifting procedure.

\section{Finding violated valid inequalities through an auxiliary integer program}

Although the method presented in the previous section can be implemented in polynomial time, it can only detect violated inequalities of a certain form (those corresponding to cycles in the conflict graph of the original graph). To detect violated inequalities that are more general, we propose a method that uses an auxiliary integer linear program. 


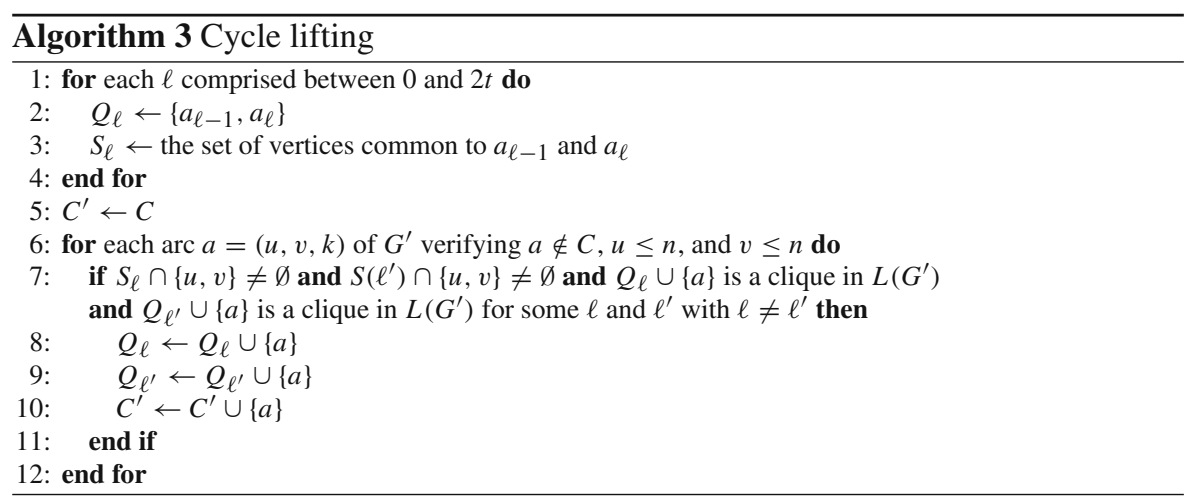

Let $\left\{\hat{X}_{i j}^{k}\right\}$ denote an optimal solution of the linear relaxation of $(P)$. There exists an integer constant $D$ such that $D \hat{X}_{i j}^{k}$ is an integer for each $(i, j, k)$. For each $k \in K$ the $D \hat{X}_{i j}^{k}$ constitute a flow starting and ending at the $k$ th depot, i.e., a monochromatic flow. By a standard argument of network flow theory, this flow can actually be expressed as a sum of incidence vectors of circuits containing the $k$ th depot. Let $\mathcal{P}^{k}$ denote the family of distinct circuits appearing in this decomposition of the monochromatic flow corresponding to the $k$ th depot. Let also $n_{p}$ denote the number of times that circuit $p$ appears in the decomposition. Finally, let us define $\mathcal{P}$ as $\bigcup_{k \in K} \mathcal{P}^{k}$ and $w_{p}$ as $n_{p} / D$ for any $p \in \mathcal{P}$.

In the previous section, we made use of the similarity between the MDVSP and the stable set problem to detect some violated odd-set inequalities. Actually, the MDVSP is also similar to the matching problem (see "Background"), in the sense that the fractional solutions of its linear relaxation remind one of the fractional solutions of the linear relaxation of the matching problem. There is a correspondence between the circuits in the family $\mathcal{P}$ defined above and the edges of an undirected graph, and a correspondence between the violated odd-set inequalities and the violated blossom inequalities of the matching polytope. Thus we define a matrix $M=\left(m_{i p}\right)$ of dimension $|V| \times|\mathcal{P}|$ whose columns are the incidence vectors of circuits in $\mathcal{P}$ and look for an odd subset $S_{1}^{\prime}$ of $\{1,2, \ldots, n\}$ such that the intersection of $S_{1}^{\prime}$ and any $p \in \mathcal{P}$ includes at most two vertices. The reason for this is that the circuits containing two vertices in $S_{1}^{\prime}$ play a role analogous to that of blossom edges in matching theory, while the set of circuits containing exactly one vertex in $S_{1}^{\prime}$ plays the role of an (odd) edge cut. Because we are looking for a violated inequality, we try to find an odd subset $S_{1}^{\prime}$ that also minimizes the sum of the $w_{p}$ such that $S_{1}^{\prime} \cap p$ is a singleton. The procedure we have just described mimics the Padberg-Rao procedure for finding violated blossom inequalities (see Padberg and Rao 1982).

To illustrate these concepts, we present a submatrix of the vertex-circuit incidence matrix corresponding to the structure displayed in Fig. 3. Table 1 contains this submatrix (the entries equal to 0 are not shown). Its first row contains the weights (multiplied by 1,000 ) of the circuits in the decomposition of the LP solution represented in Fig. 3. The other rows of Table 1 correspond to the 13 vertices in Fig. 3, respectively. The 
Table 1 The portion of the vertex-circuit incidence matrix corresponding to the nodes in Fig. 3

\begin{tabular}{|c|c|c|c|c|c|c|c|c|c|c|c|c|c|c|c|c|c|c|}
\hline & 307 & 638 & 302 & 55 & 693 & 421 & 307 & 588 & 579 & 412 & 643 & 412 & 55 & 357 & 277 & 105 & 588 & 59 \\
\hline T41 & 1 & & & & 1 & & & & & & & & & & & & & \\
\hline T163 & 1 & 1 & & & & & & & & & & & 1 & & & & & \\
\hline T316 & & 1 & 1 & 1 & & & & & & & & & & & & & & 1 \\
\hline T175 & & & 1 & & & 1 & & & & & & & & & 1 & & & \\
\hline T208 & & & 1 & 1 & & & & & & & 1 & & & & & & & \\
\hline T315 & & & & & & & & & & & 1 & & & 1 & & & & \\
\hline T160 & & & & & 1 & & 1 & & & & & & & & & & & \\
\hline T29 & & & & & & & 1 & 1 & & & & & & & & 1 & & \\
\hline T231 & & & & & & 1 & & & 1 & & & & & & & & & \\
\hline T165 & & & & & & & & 1 & & & & 1 & & & & & & \\
\hline T193 & & & & & & & & & & & & 1 & & & & & 1 & \\
\hline T66 & & & & & & & & & 1 & 1 & & & & & & & & 1 \\
\hline T216 & & & & & & & & 1 & & 1 & & & & & & & & \\
\hline
\end{tabular}

The first row contains the weights of the circuits (multiplied by 1,000). The rows in boldface type correspond to the vertices in $S_{1}^{\prime}$. The weights of circuits containing exactly one vertex in $S_{1}^{\prime}$ are also in boldface type

labels of the rows displayed in boldface type are the vertices in $S_{1}^{\prime}$. Note that in every column, there are at most two ones belonging to rows in $S_{1}^{\prime}$. The sum of the weights of the circuits whose intersection with $S_{1}^{\prime}$ is a singleton is smaller than 1 (actually it equals 0.506 ), indicating that $S_{1}^{\prime}$ is precisely the kind of odd set that we are looking for.

Note that the Inequality (4) corresponding to the structure illustrated in Fig. 3 is

$$
\begin{aligned}
X_{41,163}^{1} & +X_{163,316}^{2}+X_{208,316}^{2}+X_{208,315}^{1}+X_{175,208}^{2}+X_{175,231}^{1}+X_{66,231}^{1}+X_{66,216}^{1} \\
& +X_{165,216}^{1}+X_{165,193}^{2}+X_{29,165}^{1}+X_{29,160}^{1}+X_{41,160}^{1} \leq 6
\end{aligned}
$$

which is violated by the current solution of the linear relaxation since the sum of the flow values indicated on Fig. 3 equals 6.247. Hence, the "violation" (i.e., the difference between the sum of the flow values, on one hand, and the right-hand side of the inequality, on the other) is equal to $(1-0.506) / 2$, as in the conclusion of Proposition 2 (although this example does not quite verify the hypotheses of the proposition). Consider now a solution of the linear relaxation that is identical to the one illustrated in Fig. 3 except that $X_{208,316}^{2}$ equals 0.302, and $X_{208,209}^{2}$ equals 0.55 . In the modified solution, the arc $(208,209,2)$ is of the same color as $(175,208,2)$ and $(208,316,2)$; hence it cannot be a thorn. This explains the fact that the violation for the modified solution, which equals 0.192 , is different from $(1-0.506) / 2$.

To formulate the so-called auxiliary problem, we introduce the binary variables $x_{i}$ (for $i \in V$ ), $y_{p}$ (for $p \in \mathcal{P}$ ), and $z_{p}$ (for $p \in \mathcal{P}$ ). The variable $x_{i}$ has the value 1 if and only if $i$ belongs to $S_{1}^{\prime}, y_{p}$ the value 1 if and only if $S_{1}^{\prime} \cap p$ is nonempty, and $z_{p}$ the value 1 if and only if $S_{1}^{\prime} \cap p$ is a singleton. The variable $k$ represents the number ||$\left.S_{1}^{\prime} \mid / 2\right\rfloor$ and has been introduced to bound $\left|S_{1}^{\prime}\right|$ (from below and from above). Here is the auxiliary problem $(A P)$. Note that those $z_{p}$ that are equal to 1 play a role analogous to that of an odd cut in the Padberg-Rao procedure mentioned above. 


$$
\min \sum_{p \in \mathcal{P}} w_{p} z_{p}
$$

subject to

$$
\begin{gathered}
\sum_{i \in V} m_{i p} x_{i}=2 y_{p}-z_{p} \quad \forall p \in \mathcal{P} \\
\sum_{i \in V} x_{i}=2 k+1 \\
k_{\min } \leq k \leq k_{\max } \\
x_{i}=0 \quad \forall i>n \\
k \in \mathbb{Z}, k \geq 0 \\
x_{i}, y_{p}, z_{p} \in\{0,1\} \quad \forall i \in V, \quad \forall p \in \mathcal{P}
\end{gathered}
$$

Since the expression $\sum_{i \in V} m_{i p} x_{i}$ is nonnegative, Constraints (7) imply that $y_{p} \geq z_{p}$ holds for all $p$ in $\mathcal{P}$. Hence, $y_{p}$ equals 1 if and only if $\sum_{i \in V} m_{i p} x_{i}$ is greater than 0 , and $z_{p}$ equals 1 if and only if $\sum_{i \in V} m_{i p} x_{i}$ equals 1 . We conclude that given the constraints of $(A P), y_{p}$ and $z_{p}$ are indeed defined as above. Constraint (8) forces $S_{1}^{\prime}$ to contain an odd number of vertices. Constraints (9) have been included because putting restrictions on the value of $k$ (and hence of $\left|S_{1}^{\prime}\right|$ ) may enable us to find several violated inequalities (by assigning diverse values to the constants $k_{\min }$ and $k_{\max }$ ). Constraints (10) are necessary because we do not want $S_{1}^{\prime}$ (the set of $i$ such that $x_{i}$ equals 1 ) to contain any depot (see Definition 1).

In our opinion, both the auxiliary problem and the closely related problem of separating inequalities of type (4) are NP-complete. However, we have not been able to prove this. In any case solving $(A P)$ is time-consuming, even with the latest version of a commercial software. Furthermore, we are not necessarily looking for an optimal solution since a feasible solution of value less than 1 may give rise to a violated odd-set inequality (see below). Thus it seems reasonable to fix the values of $k_{\min }$ and $k_{\max }$, solve the resulting instance of $(A P)$, and keep track of all promising feasible solutions of $(A P)$. In our experiments, we have set $k_{\min }$ equal to 1 and $k_{\max }$ equal to $(n-|K|) / 2$.

We shall now use a feasible solution of $(A P)$ to try to find a violated odd-set inequality and lift it at the same time. We first consider the collection $\mathcal{P}^{\prime}$ of circuits $p$ such that $y_{p}=1$ and $z_{p}=0$ hold (i.e., the circuits $p$ verifying $\sum_{i \in V} m_{i p} x_{i}=2$ ). Let $i_{1}(p)$ and $i_{2}(p)$ denote the two indices $i$ with $m_{i p}=x_{i}=1$ (where $i_{2}(p)$ is further than $i_{1}(p)$ from the depot on the circuit $\left.p\right)$. Finally, we define $F_{1}$ as the set of arcs belonging to the subpath of $p$ between $i_{1}(p)$ and $i_{2}(p)$ (for any $p$ ). In most cases, $S_{1}$ (the set of vertices where all the arcs are in conflict) will coincide with $S_{1}^{\prime}$. It may happen, however, that for some circuits $p$ and $p^{\prime}$ that have the same color, $i_{2}(p)$ is identical with $i_{1}\left(p^{\prime}\right)$. For this reason, we define $S_{1}$ as follows:

$$
S_{1}=\left\{i \in S^{\prime} \mid \operatorname{col}\left(\delta_{F_{1}}^{-}(i)\right) \cap \operatorname{col}\left(\delta_{F_{1}}^{+}(i)\right)=\emptyset\right\},
$$


where $S^{\prime}$ denotes the set of all vertices incident upon arcs in $F_{1}$. Clearly, $S_{1}$ is a subset of $S_{1}^{\prime}$. We define $S_{2}$ as $S^{\prime} \backslash S_{1}$ and use Algorithm 4 to construct a submultigraph $H=(S, F)$ verifying Definition 1. Algorithm 4 processes the $\operatorname{arcs}(i, j, k)$ with a nonzero flow in the lexicographic order, i.e., it processes arc $\left(i_{1}, j_{1}, k_{1}\right)$ before arc $\left(i_{2}, j_{2}, k_{2}\right)$ if and only if one of the following conditions is satisfied:

1. $i_{1}<i_{2}$,

2. $i_{1}=i_{2}$ and $j_{1}<j_{2}$,

3. $i_{1}=i_{2}, j_{1}=j_{2}$, and $k_{1}<k_{2}$.

A source (resp. sink) in $H$ is a vertex $i$ with $\delta_{F}^{-}(i)=\emptyset\left(\operatorname{resp} . \delta_{F}^{+}(i)=\emptyset\right)$. To alleviate the notation, we let $\operatorname{col}_{F}^{+}(i)\left(\operatorname{resp} \cdot \operatorname{col}_{F}^{-}(i)\right)$ denote $\operatorname{col}\left(\delta_{F}^{+}(i)\right)$ (resp. $\operatorname{col}^{-}\left(\delta_{F}^{+}(i)\right)$ and $\operatorname{col}_{F}(i)$ denote $\operatorname{col}_{F}^{+}(i) \cup \operatorname{col}_{F}^{-}(i)$. Thus at the beginning of Algorithm $4, \operatorname{col}_{F}^{+}(i)\left(\operatorname{resp} \cdot \operatorname{col}_{F}^{-}(i)\right)$ denotes the set of colors of $\operatorname{arcs}$ in $\delta_{F}^{+}(i)\left(\operatorname{resp} . \delta_{F}^{-}(i)\right)$ that belong to the subpath of $p$ between $i_{1}(p)$ and $i_{2}(p)$ for some $p$ in $\mathcal{P}$. Observe that throughout the algorithm, a vertex $i \in S_{1}$ always satisfies the property that the arcs incident to it are in conflict. We then apply the same algorithm to process the arcs with a flow equal to 0 . If we define $S_{3}$ as the set of vertices that are incident to arcs in $F_{1} \cup F_{2}$ and do not belong to $S_{1} \cup S_{2}$, we obtain a submultigraph $H=\left(S_{1} \cup S_{2} \cup S_{3}, F_{1} \cup F_{2}\right)$ verifying Definition 1. If Inequality (4) is violated, we add it to the linear program. Since $S_{1}$ is not necessarily equal to $S_{1}^{\prime}$, the cardinality of $S_{1}$ may be even. In that case, Inequality (4) cannot be violated. It cannot be violated either if the optimal value of $(A P)$ is at least 1 . Therefore we will execute Algorithm 4 only if the optimal value of $(A P)$ is smaller than 1 . In many cases, Inequality (4) for the resulting submultigraph will be violated. Proposition 2 shows that this will be so in an important special case (which does not exhaust all possibilities).

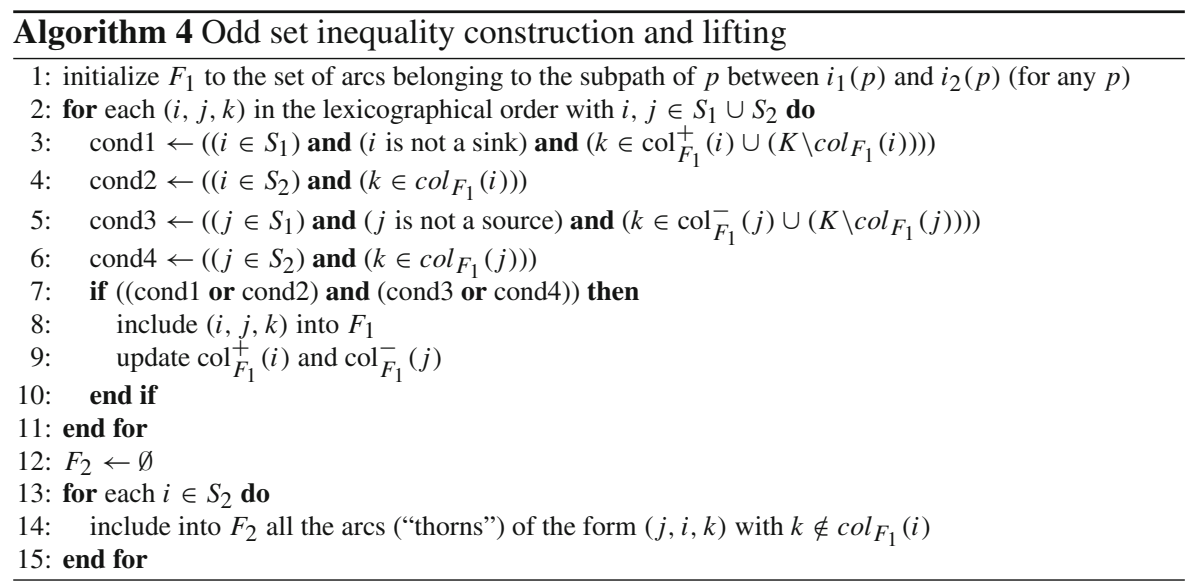

Proposition 2 Assume that the value of a feasible solution of the auxiliary problem $($ denoted by $\alpha)$ is less than 1 . As above, let $\mathcal{P}$ denote the set of paths in the decomposition of the feasible solution considered and $\mathcal{P}^{\prime}$ the set of circuits in $\mathcal{P}$ that include 
exactly 2 vertices in $S_{1}$. If $S_{1}$ equals $S_{1}^{\prime}$ and no path in $\mathcal{P} \backslash \mathcal{P}^{\prime}$ includes any arc of the submultigraph induced by $S_{1} \cup S_{2}$, then Inequality (4) for the submultigraph constructed by Algorithm 4 is violated and the corresponding violation equals $(1-\alpha) / 2$.

Proof Let $H=(S, F)$ be the submultigraph built by Algorithm 4 using the feasible solution whose objective value is less than 1 . Note that because of our assumptions, the only arcs lifted have a flow of 0 . First we evaluate the expression $\sum_{(j, i, k) \in F, i \in S_{1}} X_{j i}^{k}$ (note that we write $X_{j i}^{k}$ instead of $\hat{X}_{j i}^{k}$ to alleviate the notation). We let $\mathcal{P}^{\prime \prime}$ denote the set of circuits in $\mathcal{P}$ that include exactly one vertex in $S_{1}$. For each $p$ in $\mathcal{P}^{\prime}$, we let $i_{1}(p)$ and $i_{2}(p)$ denote the two vertices in $S_{1}$ that $p$ contains; without loss of generality, we assume that $p$ is directed from $i_{1}(p)$ to $i_{2}(p)$. Observe that any arc $(j, i, k)$ belonging to $F$ must appear on some path in $\mathcal{P}^{\prime}$ (by our assumption that no path in $\mathcal{P} \backslash \mathcal{P}^{\prime}$ includes any arc of the submultigraph induced by $S_{1} \cup S_{2}$ ). If we also assume that $i$ belongs to $S_{1}$, the vertex $i$ must be of the form $i_{2}(p)$ for some $p$ in $\mathcal{P}^{\prime}$. Therefore, we have

$$
\sum_{(j, i, k) \in F, i \in S_{1}} X_{j i}^{k}=\sum_{p \in \mathcal{P}^{\prime}} \sum_{\left(j, i_{2}(p), k\right) \in A} X_{j i_{2}(p)}^{k}=\sum_{p \in \mathcal{P}^{\prime}} w_{p} .
$$

On the other hand, we have

$$
\sum_{(j, i, k) \in A \backslash F, i \in S_{1}} X_{j i}^{k}=\sum_{p \in \mathcal{P}^{\prime}} w_{p}+\sum_{p \in \mathcal{P}^{\prime \prime}} w_{p}
$$

because $\sum_{p \in \mathcal{P}^{\prime}} w_{p}+\sum_{p \in \mathcal{P}^{\prime \prime}} w_{p}$ equals the sum of the flow values of the arcs in the directed cut $\left\{(u, v, k) \in A \mid u \notin S_{1} \cup S_{2}, v \in S_{1}\right\}$.

The following relations can be checked easily:

$$
\begin{aligned}
\left|S_{1}\right| & =\sum_{(j, i, k) \in A, i \in S_{1}} X_{j i}^{k}=\sum_{(j, i, k) \in F, i \in S_{1}} X_{j i}^{k}+\sum_{(j, i, k) \in A \backslash F, i \in S_{1}} X_{i j}^{k} \\
& =\sum_{(j, i, k) \in F, i \in S_{1}} X_{j i}^{k}+\sum_{p \in \mathcal{P}^{\prime}} w_{p}+\sum_{p \in \mathcal{P}^{\prime \prime}} w_{p} \\
& =2 \sum_{(j, i, k) \in F, i \in S_{1}} X_{j i}^{k}+\sum_{p \in \mathcal{P}^{\prime \prime}} w_{p} \\
& =2 \sum_{(j, i, k) \in F, i \in S_{1}} X_{j i}^{k}+\alpha .
\end{aligned}
$$

We conclude that

$$
\sum_{(j, i, k) \in F, i \in S_{1}} X_{j i}^{k}=\frac{\left|S_{1}\right|-\alpha}{2}=\left\lfloor\frac{\left|S_{1}\right|}{2}\right\rfloor+\frac{1-\alpha}{2} .
$$

Therefore, the proposition holds if $S_{2}$ is empty.

We now evaluate $\sum_{(j, i, k) \in F} X_{j i}^{k}$ in the case where $S_{2}$ is not empty. We have

$$
\sum_{(j, i, k) \in F} X_{j i}^{k}=\sum_{(j, i, k) \in F, i \in S_{1}} X_{j i}^{k}+\sum_{(j, i, k) \in F, i \in S_{2}} X_{j i}^{k}=\left\lfloor\frac{\left|S_{1}\right|}{2}\right\rfloor+\frac{1-\alpha}{2}+\sum_{(j, i, k) \in F, i \in S_{2}} X_{j i}^{k} .
$$


Observe that for a given $i$ in $S_{2}$, we have

$$
\sum_{(j, i, k) \in F} X_{j i}^{k}=\sum_{(j, i, k) \in F_{1}} X_{j i}^{k}+\sum_{(j, i, k) \in F_{2}} X_{j i}^{k}=\sum_{(j, i, k) \in F_{1}} X_{j i}^{k}+\sum_{(j, i, k) \in A, k \notin K(i)} X_{j i}^{k} .
$$

Let $K(i)$ denote the set of colors of the arcs incident to the vertex $i \in S_{2}$ at the beginning of Algorithm 4. Because of our assumptions, all the arcs of the form $(j, i, k)$ for $k \in K(i)$ belong to $F_{1}$ and all the arcs of the form $(j, i, k)$ for $k \notin K(i)$ are thorns. Therefore we obtain

$$
\sum_{(j, i, k) \in F} X_{j i}^{k}=\sum_{(j, i, k) \in A, k \in K(i)} X_{j i}^{k}+\sum_{(j, i, k) \in A, k \notin K(i)} X_{j i}^{k}=1 .
$$

Finally, we conclude that under the assumptions of the proposition, the following relations hold.

$$
\sum_{(j, i, k) \in F} X_{j i}^{k}=\left\lfloor\frac{\left|S_{1}\right|}{2}\right\rfloor+\frac{1-\alpha}{2}+\sum_{i \in S_{2}} \sum_{(j, i, k) \in F} X_{j i}^{k}=\left\lfloor\frac{\left|S_{1}\right|}{2}\right\rfloor+\frac{1-\alpha}{2}+\left|S_{2}\right|
$$

This completes the proof that the proposition also holds in the case where $S_{2}$ is not empty.

To illustrate the proof of Proposition 2, we consider again the middle graph in Fig. 1. In this example the vertices $a, b$, and $c$ belong to $S_{1}$ while $d$ belongs to $S_{2}$ and $e$ to $S_{3}$. The paths in $\mathcal{P}^{\prime}$ are the paths $\left(D_{1}, a, b, D_{1}\right),\left(D_{2}, b, c, D_{2}\right)$, and $\left(D_{2}, a, d, c, D_{2}\right)$. The set $\mathcal{P}^{\prime \prime}$ is empty. We observe that $\sum\left\{X_{j i}^{k} \mid(j, i, k) \in F, i \in S_{1}\right\}$ equals 1.5 (the sum of the flow values for the $\operatorname{arcs}(a, b, 1),(b, c, 2)$, and $(d, c, 2))$; hence it is equal to $\sum_{p \in \mathcal{P}^{\prime}} w_{p}$. In the same fashion, $\sum\left\{X_{j i}^{k} \mid(j, i, k) \in A \backslash F, i \in S_{1}\right\}$ equals 1.5 [the sum of the flow values for the $\operatorname{arcs}\left(D_{1}, a, 1\right),\left(D_{2}, a, 2\right)$, and $\left.\left(D_{2}, b, 2\right)\right]$; hence it is equal to $\sum_{p \in \mathcal{P}^{\prime}} w_{p}+\sum_{p \in \mathcal{P}^{\prime \prime}} w_{p}$. To compute the flow on all the arcs of the conflictual submultigraph (i.e., the submultigraph induced by $\{a, b, c, d, e\}$ ), we must include the flow on $\operatorname{arcs}(a, d, 2)$ and $(e, d, 1)$, that is, the amount that flows into $d$. We observe that $\sum\left\{X_{j i}^{k} \mid(j, i, k) \in F, i \in S_{1}\right\}+X_{a d}^{2}+X_{e d}^{1}$ equals $1.5+1.0=2.5$, which shows that Inequality (4) is violated by an amount of 0.5 . To conclude we note that even if the assumptions of Proposition 2 are not satisfied, its conclusion may be true (see the example of the thorny cycle in Fig. 3, which we have already discussed).

\section{Variable fixing and strategies}

Any method for solving the MDVSP by a branch-and-bound or branch-and-cut algorithm can be "improved" by fixing some variables to 0 before sophisticated techniques are used. To do this, we must consider the dual of the linear relaxation of $(P)$, which we denote $(D L P)$. For $i$ comprised between 1 and $n$, let $\pi_{i}$ denote the dual variable corresponding to Constraint (1) for vertex $i$. Similarly, for $k \in K$, let $\pi_{n+k}$ denote the dual variable corresponding to Constraint (2). Finally, let $\rho_{i}^{k}$ denote the dual variable corresponding to Constraint (3) for color $k$ and vertex $i$. After multiplying each of 
the Constraints (1) by -1 and changing the sign of each of the Constraints (2) (i.e., replacing $\sum_{j=1}^{n} X_{n+k, j}^{k} \leq v_{k}$ by $-\sum_{j=1}^{n} X_{n+k, j}^{k} \geq-v_{k}$ ), one can easily verify that (DLP) is the following linear program.

$$
\max -\sum_{i=1}^{n} \pi_{i}-\sum_{k \in K} v_{k} \pi_{n+k}
$$

subject to

$$
\begin{aligned}
-\pi_{i}-\rho_{i}^{k}+\rho_{j}^{k} \leq c_{i j} & \text { for all }(i, j, k) \in A \\
\pi_{n+k} \geq 0 & \text { for } k \in K \\
\rho_{n+k}^{k}=0 & \forall k \in K
\end{aligned}
$$

Note that we may set $\rho_{n+k}^{k}$ to 0 because the system formed by Constraints (3) is not of full rank. The reduced cost of the variable $X_{i j}^{k}$ [in the linear relaxation of $(P)$ ] is of course given by the formula $c_{i j}+\pi_{i}+\rho_{i}^{k}-\rho_{j}^{k}$, and it is possible to use the values of dual variables in an optimal solution returned by CPLEX (say) to compute the reduced costs of the $X_{i j}^{k}$ (we will call this the trivial method). There are many optimal dual solutions, however, and it is possible to compute reduced costs that are "better" from the point of view of variable fixing. We now outline the bidirectional method introduced by Irnich et al. (2010).

Constraints (11) can be rewritten as

$$
\rho_{j}^{k} \leq \rho_{i}^{k}+\pi_{i}+c_{i j} \quad \text { for all }(i, j, k) \in A
$$

It is easy to show, by using mathematical induction, that $\rho_{j}^{k}$ is at most the length of a shortest path from $D_{k}$ to $j$ in the network $\left(V, A^{\prime}, c^{\prime}\right)$, where $A^{\prime}$ is the set of couples $(i, j)$ such that $(i, j, k)$ belongs to $A$ for at least one $k$ and the vector $c^{\prime}$ is defined by $c_{i j}^{\prime}=\pi_{i}+c_{i j}$ for all $(i, j, k)$. In a similar fashion, $-\rho_{j}^{k}$ is at most the length of a shortest path from $j$ to $D_{k}$ in the network $\left(V, A^{\prime}, c^{\prime}\right)$. For a given vertex $i$, let $\lambda_{i}$ (resp. $\lambda_{i}^{\prime}$ ) denote the length of a shortest path from $D_{k}$ to $i$ (resp. from $i$ to $D_{k}$ ) in the network $\left(V, A^{\prime}, c^{\prime}\right)$. For any arc $(i, j, k)$ there exists an optimal dual solution in which $\rho_{i}^{k}$ equals $\lambda_{i}$ and $-\rho_{j}^{k}$ equals $\lambda_{j}^{\prime}$. Hence we may take $c_{i j}+\pi_{i}+\lambda_{i}+\lambda_{j}^{\prime}$ as the value of the reduced cost of variable $X_{i j}^{k}$. The bidirectional method consumes more time than the trivial method because it requires solving a shortest path problem in both directions for each $D^{k}$. On the other hand, it enables one to find, for each arc, a dual solution guaranteeing the largest possible reduced cost.

Variable fixing requires not only reduced costs, but an algorithm that produces a heuristic solution. We now describe such an algorithm. Clearly, an MDVSP instance could be solved quickly if $k$ were equal to 1 . Indeed in that case, the MDVSP reduces to a minimum-cost flow problem. Given an MDVSP instance defined by a multigraph $G$, an availability vector $v$, and a cost vector $c$, we define an instance with a single depot by replacing the $k$ depots by a single one (with a label equal to $n+1$ and an 
availability equal to $\sum_{k \in K} v_{k}$ ) and redefining the costs of the arcs incident upon the depot as follows:

$c_{n+1, i}^{\prime}=\min \left\{c_{n+k, i} \mid k \in K\right\}, \quad c_{i, n+1}^{\prime}=\min \left\{c_{i, n+k} \mid k \in K\right\}, \quad c_{i j}^{\prime}=c_{i j} \quad$ for all $i, j \leq n$.

Let $(P S)$ denote the multicommodity flow formulation of this single-depot problem. Since minimum-cost flow problems have the integrality property, we need not use a branch-and-bound algorithm to solve $(P S)$. Solving $(P S)$ by the simplex method will give us reduced costs for the variables. We then sort the variables in increasing order of reduced costs and choose the first $q_{1}$ variables in that order. Let $A^{\prime}$ denote the set of arcs corresponding to these variables. We define the first reduced instance [denoted by $(P R l)]$ as the restriction of $(P)$ to $A^{\prime}$, solve the linear relaxation of $(P R l)$, choose the $q_{2}$ variables (or arcs) with the smallest reduced costs in that relaxation, and define $(P R 2)$ as the restriction of $(P)$ to these $q_{2}$ variables. The heuristic solution is the solution of the integer program (PR2) and its value will be denoted by $U B$.

One must be able to compute the heuristic solution much faster than the optimal solution of the MDVSP instance. Thus $q_{1}$ and $q_{2}$ must not be too large. Let $m$ denote the number of arcs and NbSmallCost PS (resp. NbSmallCost PR1) the number of variables in $(P S)$ [resp. $(P R l)]$ whose reduced cost is smaller than 0.1 . We define $q_{1}$ and $q_{2}$ as follows.

$$
\begin{aligned}
& \operatorname{VarMax} 1=\max \{\lfloor 10 n\rfloor+1,\lfloor m / 10\rfloor+1\}, \quad q_{1}=\min \{\text { VarMax } 1, \text { NbSmallCost } P S\} \\
& \operatorname{VarMax} 2=\max \{\lfloor n\rfloor+1,\lfloor m / 10\rfloor+1\}, \quad q_{2}=\min \{\operatorname{VarMax} 2, N \text { S SmallCost } P R 1\}
\end{aligned}
$$

We apply the following proposition to fix some variables of $(P)$ to 0 . For a more general statement, we refer the reader to Proposition 3.1 in Hadjar et al. (2006).

Proposition 3 Assume that the reduced cost of some variable $X_{i j}^{k}$ of $(P)$ is at least $U B-O P T$, where $O P T$ denotes the optimal value of the linear relaxation of $(P)$. Let $\hat{X}$ denote a feasible solution of $(P)$ whose value is strictly less than $U B$ (if it exists). Then $\hat{X}_{i j}^{k}$ equals 0 .

We use Algorithm 5 to eliminate variables (i.e., to assign the value 0 to them). It relies on Proposition 3. Note that we first apply the trivial method to eliminate as many variables as possible. Once this first group of variables have been eliminated, the application of the bidirectional method is not as costly as it would be otherwise, since the networks $D_{k}$ have far fewer arcs than before.

We now describe the strategies that we compared during our experiments. We start by presenting an algorithm for strengthening the linear programming relaxation of $(P)$, the multicommodity flow model for an MDVSP instance. Algorithm 6 is actually a template because it does not specify the family of violated inequalities to be considered.

There are three families of inequalities to consider: the inequalities found by the method in "Finding violated valid inequalities through a search of the conflict graph" (the odd-cycle inequalities), those found by the method in "Finding violated valid inequalities through an auxiliary integer program" (the odd-set inequalities), and the inequalities corresponding to cliques of size 3 (the 3 -clique inequalities). Note that 

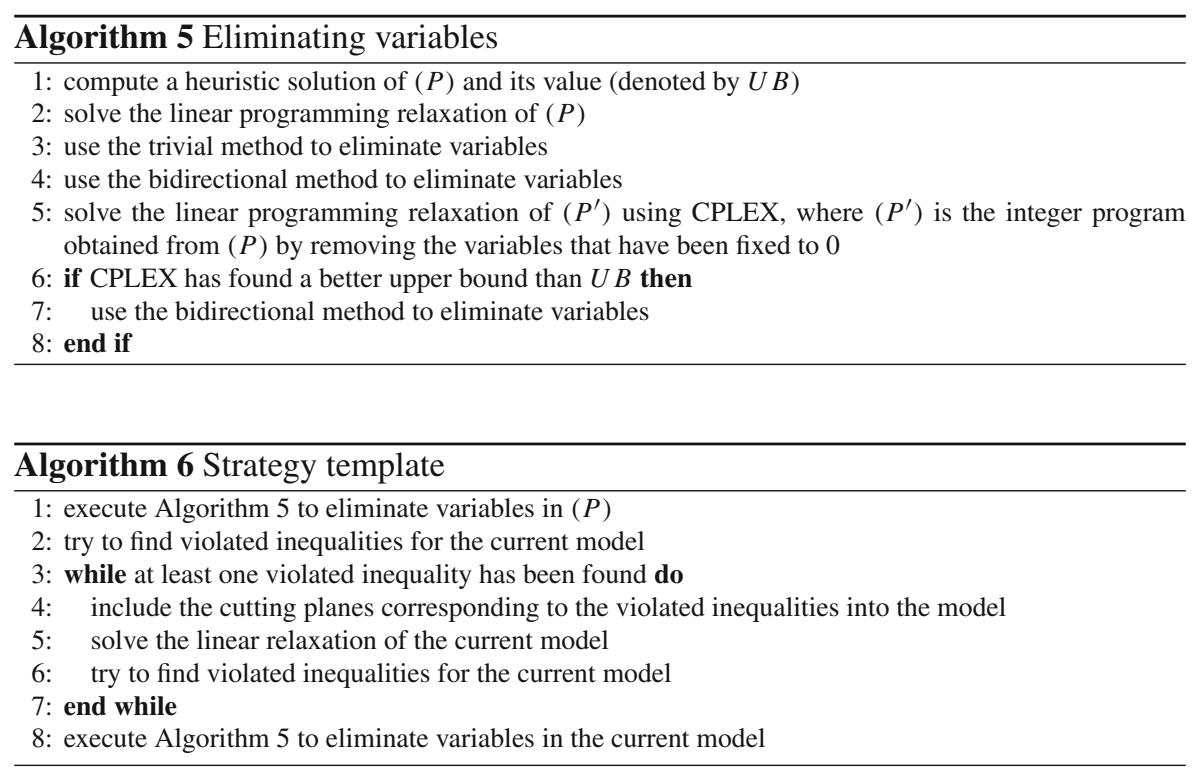

3-clique inequalities are a subfamily of odd-cycle inequalities since a 3-clique is also a cycle of length 3 . Because enumerating 3-cliques does not consume much time, we decided to look for violated 3-clique inequalities through enumeration rather than resorting to the more general separation algorithm described in "Finding violated valid inequalities through a search of the conflict graph". In that section we defined $G^{\prime}$ as the support of the optimal solution of the linear relaxation and denoted the conflict graph of $G^{\prime}$ by $L\left(G^{\prime}\right)$. Because the conflict graph is quite large and the detection of odd cycles takes a long time, we have redefined the arc set of $G^{\prime}$ as $A^{\prime}=\left\{(i, j, k) \mid X_{i j}^{k}>\right.$ $0.125\}$ in our experiments. When attempting to find violated odd-cycle (resp. odd-set) inequalities, we only retain those for which the violation is at least equal to 0.2 (resp. $10^{-3}$ ). On the other hand, we retain all 3 -clique inequalities that are violated by the current solution. We tested the seven strategies listed below. Note that when we refer to CPLEX (within Strategies 1-7), we mean the version of the CPLEX mixed-integer programming solver with depth-first search and no "default" cutting planes.

1. Strategy 1 consists of running Algorithm 5 for eliminating variables and solving the current model with CPLEX.

2. Strategy 2 consists of running Algorithm 6 while looking for odd-cycle inequalities, to tighten the LP relaxation. The MIP option of CPLEX is then called to complete the solution process (without adding new cutting planes at the root node or any of its descendants).

3. Strategy 3 is identical to Strategy 2 except that we look only for violated odd-set inequalities.

4. Strategy 4 is identical to Strategy 2 except that we look only for violated 3 -clique inequalities. 
5. Strategy 5 is identical to Strategy 2 except that whenever we look for violated inequalities, we look for violated odd-cycle inequalities, violated odd-set inequalities, and violated 3-clique inequalities.

6. Strategy 6 consists of running Algorithm 6 while looking for the three types of violated inequalities at each iteration (as in Strategy 5), to tighten the LP relaxation. The MIP option of CPLEX is then called to complete the solution process while adding cutting planes corresponding to violated odd-cycle inequalities at each descendant of the root node.

7. Strategy 7 is identical to Strategy 6 except that to tighten the LP relaxation, only violated odd-set and 3-clique inequalities are considered.

Note that in the cases of Strategies 5 and 6, we look for all types of violated inequalities simultaneously, i.e., we look for violated odd-cycle, odd-set, and 3-clique inequalities before the loop in Algorithm 6 and at each iteration of the while loop. The same remark can be made about Strategy 7.

In our experiments we compared all seven strategies between themselves and with CPLEX. In what follows Strategy 0 denotes CPLEX with the default parameters (i.e., best-bound branching rule, CPLEX standard cutting planes, and CPLEX preprocessing phase). The preprocessing phase may or may not involve the fixing of some variables. To ascertain the value of variable fixing (as opposed to that of our cutting planes), we also decided to compare Strategies 0 to 7 with the following strategies.

8. CPLEX with variable fixing (as described in the current section), the best-bound branching rule, and the CPLEX standard cutting planes.

9. CPLEX with variable fixing (as described in the current section) and the best-bound branching rule (but no cutting planes).

10. Strategy 2 without variable fixing.

11. Strategy 5 without variable fixing.

\section{Computational results}

To test our strategies, we used a generator of pseudo-random instances that was introduced by Carpaneto et al. (1989) and used by several authors (e.g., Ribeiro and Soumis 1994; Fischetti et al. 2001; Hadjar et al. 2006). There are two types of instances: Type A instances and Type B instances. To describe a class of instances we use expressions such as 400A4, which refers to instances of Type A with 400 nodes (or trips) and four depots. We ran our programs with CPLEX 12.4.0.0 on the Briarée computer of RQCHP (the Québec High-Performance Computing Network). Briarée consists of 630 nodes, each of which includes 2 Intel processors (Intel Xeon X5650 2.66GHz Westmere-EP). The peak computing power of Briarée is 80.4 TFlops. We did not use the parallel version of CPLEX.

In all of our tables, an entry represents the average of a certain outcome over five random instances in the same class. For instance, we report in Table 2 that the average percentage of variables fixed by Strategy 2 equals $93.70 \%$ for instances in the class 600A5. To compare the strategies and assess the impact of variable fixing, we started by considering five randomly generated Type A instances with 600 nodes and five 
Table 2 Comparison of strategies for five Type A instances with 600 nodes and five depots and five Type A instances with 800 instances and five depots

\begin{tabular}{llcclcl}
\hline Strategy & $\begin{array}{l}\text { NbVarFix as } \% \\
600 \text { nodes }\end{array}$ & $\begin{array}{l}\text { NbCuts } \\
600 \text { nodes }\end{array}$ & $\begin{array}{l}\text { Total CPU }(\mathrm{s}) \\
600 \text { nodes }\end{array}$ & $\begin{array}{l}\text { NbVarFix as } \% \\
800 \text { nodes }\end{array}$ & $\begin{array}{l}\text { NbCuts } \\
800 \text { nodes }\end{array}$ & $\begin{array}{l}\text { Total CPU (s) } \\
800 \text { nodes }\end{array}$ \\
\hline 0 & 0.00 & 0.0 & $1,470.92$ & 0.00 & 0.0 & $6,041.7$ \\
1 & 92.44 & 0.0 & 630.84 & 78.23 & 0.0 & $6,615.6$ \\
2 & 93.70 & 28.0 & 480.14 & 78.79 & 30.8 & $3,358.3$ \\
3 & 93.74 & 6.2 & 620.76 & 78.33 & 1.8 & $6,307.9$ \\
4 & 92.96 & 0.4 & 625.52 & 78.23 & 0.2 & $9,428.7$ \\
5 & 93.02 & 33.8 & 511.60 & 78.22 & 41.1 & $2,985.5$ \\
6 & 94.38 & 94.8 & 682.42 & 78.22 & 131.0 & $5,238.9$ \\
7 & 93.14 & 70.8 & 621.10 & 78.33 & 90.0 & $6,146.5$ \\
8 & 92.44 & 0.0 & 640.89 & 78.23 & 0.0 & $5,105.9$ \\
9 & 92.44 & 0.0 & 857.96 & 78.23 & 0.0 & $6,666.4$ \\
10 & 0.00 & 27.2 & $1,716.44$ & 0.00 & 29.2 & $9,714.2$ \\
11 & 0.00 & 28.4 & $1,673.22$ & 0.00 & 37.4 & $11,428.9$ \\
\hline
\end{tabular}

The average number of variables for instances with 600 (resp. 800) nodes is 548417 (resp. 969901). Strategies 0 to 11 are described at the end of "Variable fixing and strategies"

depots and five randomly generated Type A instances with 800 nodes and five depots. The results of this comparison are displayed in Table 2. We first note that for the five instances with 600 nodes, Strategy 2 is the best among Strategies 1 to 7 and that the average time consumed by Strategy 2 is $25 \%$ lower than that consumed by Strategy 8 (involving our variable fixing procedure, the best-bound branching rule, and the CPLEX standard cutting planes). In the case of instances with 800 nodes, Strategy 5 is better than Strategy 2 and consumes less than $60 \%$ of the time consumed by Strategy 8 . Note also that for instances with 800 nodes, Strategy 1 consumes more time than Strategy 0; this is probably due to the efficiency of the CPLEX preprocessing phase for this particular group of instances.

For our other experiments, we considered 130 randomly generated instances, that is, five instances in the class 400A4, five instances in the class 400B4, five instances in the class 400A5, five instances in the class 400B5, and so on until instances with 900 nodes, to which we added five instances in the class 1000A4 and five instances in the class 1000B4. We set a time limit of $48 \mathrm{~h}$ for the solution of these instances by CPLEX (Strategy 0) or any of the other strategies. Two instances in the class 900B5 and one instance in each of the classes 900B4, 1000A5, 1000B4, and 1000B5 could not be solved by CPLEX within $48 \mathrm{~h}$. Of these instances, Strategy 2 could solve one instance in the class 900B5 (within $48 \mathrm{~h}$ ) and the instance in the class 1000B4 (within $48 \mathrm{~h}$ ); hence one 900B4 instance, one 900B5 instance, one 1000A5 instance, and one 1000B5 instance could not be solved (within 48 h) by Strategy 2. Strategy 5 could solve all of the instances except one 900B4 instance (not solved by either CPLEX or Strategy 2) and one 1000A4 instance. In what follows the results for the unsolved instances are not included in the statistics. 


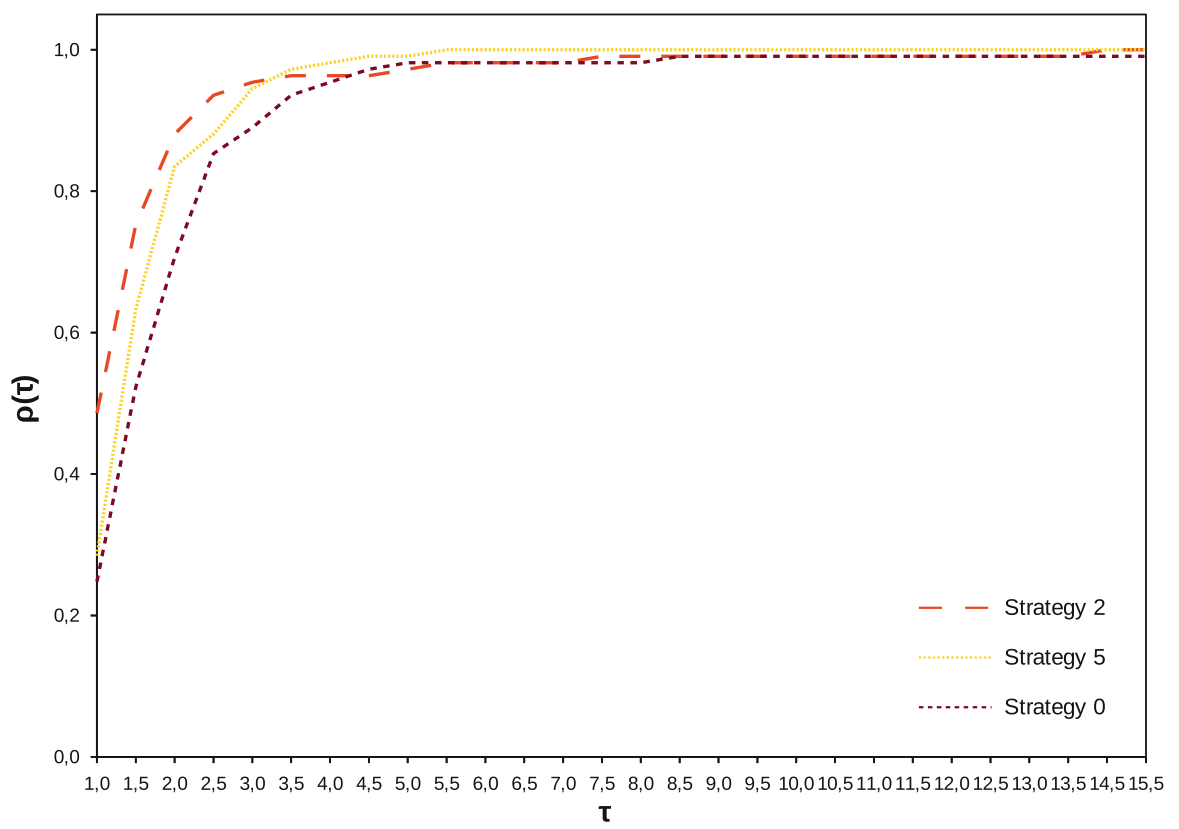

Fig. 6 Performance profiles of Strategies 0, 2, and 5

Performance profiles were introduced by Dolan and Moré (2002) and we have used them to evaluate our strategies. We let $t_{i s}$ denote the CPU time needed for solving the $i$ th instance with Strategy $s$ and define the performance ratio $r_{i s}$ as

$$
r_{i s}=\frac{t_{i s}}{\min _{s}\left\{t_{i s}\right\}} \text {. }
$$

For a real number $\tau$ at least equal to $1.0,\left\{i \mid r_{i s} \leq \tau\right\}$ denotes the set of instances for which Strategy $s$ has a "good" performance ratio (with respect to $\tau$ ). Finally, we define the performance of Strategy $s$ as the function $\rho_{s}$ :

$$
\rho_{s}(\tau)=\frac{\left|\left\{i \mid r_{i s} \leq \tau\right\}\right|}{N}
$$

where $N$ (130, in the present case) denotes the number of instances. For example, a value of 0.8 for $\rho_{s}(1.5)$ would mean that $80 \%$ of the instances can be solved by Strategy $s$ in a time that is within $150 \%$ of the best time observed for all strategies. In Fig. 6, we compare Strategies 0, 2, and 5. Strategy 2 has the best performance for small values of $\tau$; indeed, its performance profile is above the other profiles for (relatively) small values of $\tau$. Also, Fig. 6 shows that Strategy 5 converges more rapidly than the two other strategies.

We also decided to look at various instance sizes to have a more precise picture of the situation. Figure 7 displays the average time needed to solve an instance of a given size, as a function of its size. For instances of size 800 or less, Strategy 2 consumes less time than Strategy 5, while for instances of size 900 or 1,000, Strategy 5 consumes 


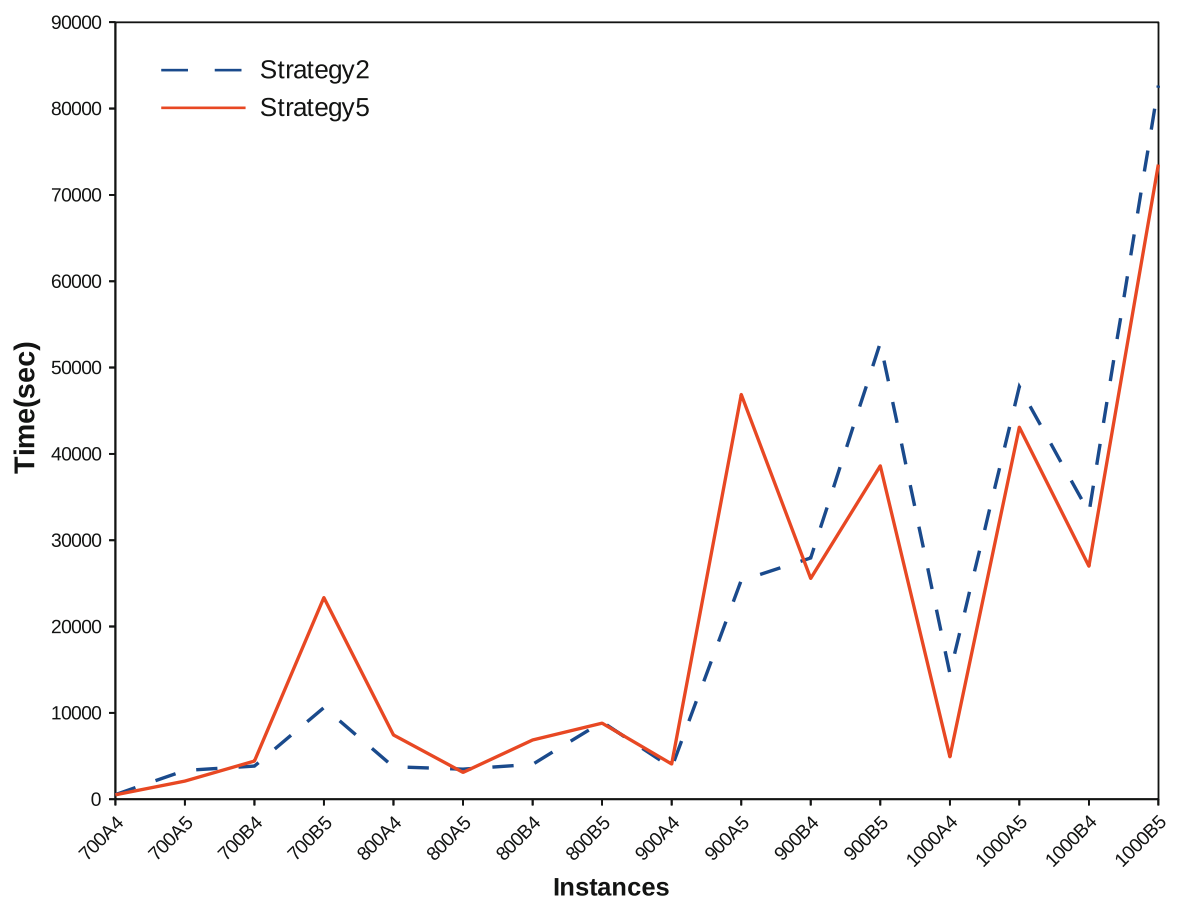

Fig. 7 Comparison of solution times for Strategies 2 and 5

Table 3 Comparison of the number of variables fixed in Strategy 2 with the number fixed in Strategy 5

\begin{tabular}{llll}
\hline $\begin{array}{l}\text { Instance } \\
\text { class }\end{array}$ & NbVar & $\begin{array}{l}\text { NbVarFix (\%) } \\
\text { Strategy 2 }\end{array}$ & $\begin{array}{l}\text { NbVarFix (\%) } \\
\text { Strategy 5 }\end{array}$ \\
\hline 400A4 & $194,452.0$ & 98.5 & 98.4 \\
400A5 & $245,253.0$ & 86.1 & 87.6 \\
500A4 & $303,904.0$ & 96.5 & 95.0 \\
500A5 & $379,906.0$ & 86.6 & 92.4 \\
600A4 & $433,015.2$ & 96.2 & 94.2 \\
600A5 & $548,417.0$ & 93.7 & 94.4 \\
700A4 & $595,441.6$ & 90.7 & 90.7 \\
700A5 & $747,081.0$ & 62.7 & 62.6 \\
800A4 & $781,039.2$ & 76.9 & 76.9 \\
800A5 & $969,901.0$ & 78.8 & 78.2 \\
900A4 & $969,084.8$ & 91.2 & 91.2 \\
900A5 & $1,231,693.0$ & 85.6 & 85.7 \\
1000A4 & $1,213,471.6$ & 77.8 & 77.6 \\
1000A5 & $1,518,470.0$ & 92.9 & 92.9 \\
\hline
\end{tabular}

less time. We conclude that looking for cutting planes based on odd sets is useful when solving large instances. We have not included into Fig. 7 the average solution times for Type A or B instances with 400, 500, or 600 trips, because those times are very 
Table 4 Numbers of cuts added in Strategies 2 and 5. Note that all cuts in Strategy 2 arise from odd cycles

\begin{tabular}{llrllllr}
\hline $\begin{array}{l}\text { Instance } \\
\text { class }\end{array}$ & $\begin{array}{l}\text { TotalNbcuts } \\
\text { Strategy 2 }\end{array}$ & $\begin{array}{l}\text { NbBNodes } \\
\text { Strategy 2 }\end{array}$ & $\begin{array}{l}\text { NbOddCycles } \\
\text { Strategy 5 }\end{array}$ & $\begin{array}{l}\text { Nb3cliques } \\
\text { Strategy 5 }\end{array}$ & $\begin{array}{l}\text { NbOddSets } \\
\text { Strategy 5 }\end{array}$ & $\begin{array}{l}\text { TotalNbcuts } \\
\text { Strategy 5 }\end{array}$ & $\begin{array}{l}\text { NbBNodes } \\
\text { Strategy 5 }\end{array}$ \\
\hline 400A4 & 14.8 & 74.4 & 16.0 & 0.0 & 6.6 & 22.6 & 88.6 \\
400A5 & 14.2 & 729.4 & 13.8 & 0.0 & 5.0 & 18.8 & 612.0 \\
500A4 & 30.0 & 592.2 & 32.2 & 0.2 & 6.6 & 39.0 & 937.6 \\
500A5 & 16.4 & 744.6 & 22.8 & 0.6 & 5.4 & 28.8 & 380.0 \\
600A4 & 34.4 & 562.6 & 31.8 & 0.2 & 3.6 & 35.6 & 425.0 \\
600A5 & 28.0 & $6,133.4$ & 26.6 & 0.6 & 4.6 & 31.8 & $7,546.6$ \\
700A4 & 31.8 & $3,637.6$ & 32.2 & 0.0 & 4.4 & 36.6 & $1,356.4$ \\
700A5 & 32.6 & $34,686.8$ & 28.6 & 0.0 & 4.4 & 33.0 & $13,453.2$ \\
800A4 & 39.2 & $30,543.0$ & 39.6 & 0.0 & 5.2 & 44.8 & $59,090.6$ \\
800A5 & 30.8 & $26,246.4$ & 35.4 & 0.2 & 5.8 & 41.4 & $18,167.8$ \\
900A4 & 24.8 & $32,643.2$ & 24.8 & 0.4 & 2.8 & 28.0 & $37,977.4$ \\
900A5 & 31.0 & $165,496.8$ & 36.6 & 0.2 & 6.4 & 43.2 & $300,869.0$ \\
1000A4 & 31.75 & $71,439.3$ & 31.0 & 0.3 & 4.5 & 35.75 & $34,021.0$ \\
1000A5 & 37.0 & $487,212.75$ & 33.75 & 0.3 & 6.75 & 40.75 & $129,831.0$ \\
\hline
\end{tabular}

small. Indeed, the average solution time for these instances is $398 \mathrm{~s}$ and the average times vary between 37 and $813 \mathrm{~s}$, except for the average times for instances in the class 600B5, which are 2,080 s for Strategy 2 and 2,186 s for Strategy 5.

The last three tables contain statistics on our results for Strategies 2 and 5. Note that we present results for Type A instances only, since the results for Type B instances are very similar to those for Type A. Table 3 contains the average percentages of variables that are fixed by each strategy. There are few differences between the two strategies, but one observes that the percentage of fixed variables (which is very high for "small" instances) decreases as the size of the instance increases. This may be due to a decrease in the quality of the upper bound (i.e., the value of the best integral solution found so far). Table 4 contains the average numbers of cutting planes for both strategies (recall that Strategy 2 only looks for cutting planes based on odd cycles). Clearly, there are very few cutting planes based on 3-cliques. Also, we observe that Strategy 5 generates more cutting planes than Strategy 2; as we saw in Fig. 7, this strategy seems to be especially useful for large instances. Table 4 also displays the average number of branching nodes (NbBNodes) for both strategies. We observe that for the instances with 1,000 trips, the number of nodes generated by Strategy 5 is significantly smaller than that generated by Strategy 2.

Finally, Table 5 contains statistics on the solutions of the auxiliary integer program (used to find cutting planes based on odd sets). As mentioned in "Finding violated valid inequalities through an auxiliary integer program", the solution of the auxiliary program does not always yield a cutting plane based on an odd set. One possibility is that the actual set is an even set; the average number of times when this event occurs is displayed in the column labelled NbEvenSets. For example, if we consider instances in the class $400 \mathrm{~A} 4$, we observe that the auxiliary program found an average of 6.6 
Table 5 Solutions of the auxiliary integer program in Strategy 5

\begin{tabular}{llllll}
\hline $\begin{array}{l}\text { Instance } \\
\text { class }\end{array}$ & NbOddSets & $\begin{array}{l}\text { NbOddSets } \\
\text { as \% }\end{array}$ & NbEvenSets & $\begin{array}{l}\text { NbEvenSets } \\
\text { as \% }\end{array}$ & NbSetsFound \\
\hline 400A4 & 6.6 & 48.5 & 0.6 & 4.4 & 13.6 \\
400A5 & 5.0 & 39.1 & 2.8 & 21.9 & 12.8 \\
500A4 & 6.6 & 36.3 & 2.0 & 11.0 & 18.2 \\
500A5 & 5.4 & 37.5 & 1.4 & 9.7 & 14.4 \\
$600 \mathrm{~A} 4$ & 3.6 & 32.1 & 0.8 & 7.1 & 11.2 \\
600A5 & 4.6 & 37.7 & 1.2 & 9.8 & 12.2 \\
700A4 & 4.4 & 31.9 & 1.2 & 8.7 & 13.8 \\
$700 \mathrm{~A} 5$ & 4.4 & 37.3 & 0.4 & 3.4 & 11.8 \\
$800 \mathrm{~A} 4$ & 5.2 & 36.1 & 0.6 & 4.2 & 14.4 \\
$800 \mathrm{~A} 5$ & 5.8 & 37.7 & 0.6 & 3.9 & 15.4 \\
$900 \mathrm{~A} 4$ & 2.8 & 26.4 & 0.4 & 3.8 & 10.6 \\
$900 \mathrm{~A} 5$ & 6.4 & 33.7 & 0.4 & 2.1 & 19 \\
1000A4 & 4.5 & 39.1 & 0.25 & 2.2 & 11.5 \\
1000A5 & 6.75 & 40.3 & 1.25 & 7.5 & 16.75 \\
\hline
\end{tabular}

cutting planes based on odd sets, an average of 0.6 solution corresponding to an even set, and an average of 6.4 solutions corresponding to an odd set whose associated inequality is not violated. Generally speaking, almost $40 \%$ of solutions found by the auxiliary program were useful, i.e., yielded cutting planes.

\section{Conclusion}

In this article, we have shown that the introduction of cutting planes based on odd cycles and odd sets enables one to decrease by a significant amount the time needed to solve an MDVSP instance. While cutting planes based on odd cycles can be found in polynomial time, the separation problem for odd sets can be formulated (in a heuristic fashion) as an integer program whose complexity is not yet known. In a similar fashion, we still do not know whether the separation problem itself is NP-complete (in spite of strong evidence to that effect). From a practical point of view, it would be desirable to propose heuristics for solving the odd-set separation problem.

Acknowledgments The work of Guy Desaulniers and Odile Marcotte was supported by their respective grants from the Natural Sciences and Engineering Research Council of Canada (NSERC). The authors are grateful to the anonymous referees for their many useful comments and suggestions.

\section{References}

Bertossi AA, Carraresi P, Gallo G (1987) On some matching problems arising in vehicle scheduling models. Networks 17:271-281

Bianco L, Mingozzi A, Ricciardelli S (1994) A set partitioning approach to the multiple depot vehicle scheduling problem. Optim Methods Softw 3:163-194 
Bodin L, Rosenfield D, Kydes A (1978) UCOST: a Micro approach to a transit planning problem. J Urban Anal 5:46-69

Carpaneto D, Dell'Amico M, Fischetti M, Toth P (1989) A branch and bound algorithm for the multiple vehicle scheduling problem. Networks 19:531-548

Caprara A, Fischetti M (1996) \{0,1/2\}-Chvátal-Gomory cuts. Math programm 74:221-235

Desaulniers G, Hickman MD (2007) Public Transit. In: Barnhart C, Laporte G (eds) Handbooks in OR \& MS: transportation, vol 14. Elsevier, Amsterdam, pp 69-127

Desrosiers J, Dumas Y, Solomon M, Soumis F (1995) Time constrained routing and scheduling. In: Ball MO, Magnanti TL, Monma CL, Nemhauser GL (eds) Handbooks in OR \& MS: network routing, vol 8. Elsevier, Amsterdam, pp 35-139

Dolan EE, Moré JJ (2002) Benchmarking optimization software with performance profiles. Math Program 91:201-213

Fischetti M, Lodi A, Martello S, Toth P (2001) A polyhedral approach to simplified crew scheduling and vehicle scheduling problems. Manag Sci 47:833-850

Gintner V, Kliewer N, Suhl L (2005) Solving large multiple-depot multiple-vehicle-type bus scheduling problems in practice. OR Spectr 27:507-523

Hadjar A, Marcotte O, Soumis F (2006) A branch-and-cut algorithm for the multiple depot vehicle scheduling problem. Oper Res 54:130-149

Irnich S, Desaulniers G, Desrosiers J, Hadjar A (2010) Path-reduced costs for eliminating arcs in routing and scheduling. INFORMS J Comput 22:297-313

Kliewer N, Mellouli T, Suhl L (2006) A time-space network based exact optimization model for multi-depot bus scheduling. Eur J Oper Res 175:1616-1627

Laurent B, Hao J-K (2009) Iterated local search for the multiple depot vehicle scheduling problem. Comput Ind Eng 57:277-286

Löbel A (1997) Optimal vehicle scheduling in public transit. PhD thesis, Technische Universität Berlin, Berlin

Löbel A (1998) Vehicle scheduling in public transit and Lagrangean Pricing. Manag Sci 44:1637-1649

Nemhauser GL, Wolsey LA (1988) Integer and combinatorial optimization. Wiley, New York

Nemhauser GL, Sigismondi G (1992) A strong cutting plane/branch-and-bound algorithm for node packing. J Oper Res Soc 43:443-457

Oukil A, Ben Amor H, Desrosiers J, El Gueddari H (2007) Stabilized column generation for highly degenerate multiple-depot vehicle scheduling problems. Comput OR 34:817-834

Padberg MW, Rao MR (1982) Odd minimum cut-sets and $b$-matchings. Math Oper Res 7:67-80

Pepin A-S, Desaulniers G, Hertz A, Huisman D (2009) Comparison of Heuristic approaches for the multiple depot vehicle scheduling problem. J Sched 12:17-30

Pulleyblank WR, Edmonds J (1974) Facets of 1-matching polyhedra. In: Berge C, Ray-Chaudhuri D (eds) Hypergraph seminar. Springer, Berlin, pp 214-242

Rebennack S (2006) Maximum stable set problem: a branch \& cut solver. Diploma Thesis, RuprechtKarls-Universität Heidelberg, Heidelberg

Ribeiro C, Soumis F (1994) A column generation approach to the multiple depot vehicle scheduling problem. Oper Res 42(41-52):1994 